\title{
Potassic-Hastingsite from the Kedrovy District (East Siberia, Russia): Petrographic Description, Crystal Chemistry, Spectroscopy, and Thermal Behavior
}

\author{
Ekaterina Kaneva ${ }^{1,2, *(\mathbb{D}}$, Tatiana Radomskaya ${ }^{1,2}$, Roman Shendrik ${ }^{1} \mathbb{D}$, Victor Chubarov ${ }^{1}$ and Victoria Danilovsky $^{3}$ \\ 1 Vinogradov Institute of Geochemistry, Siberian Branch of the Russian Academy of Sciences, \\ 664033 Irkutsk, Russia; taniaojigova@mail.ru (T.R.); r.shendrik@gmail.com (R.S.); chubarov@igc.irk.ru (V.C.) \\ 2 Department of Subsoil Use, Irkutsk National Research Technical University, 664074 Irkutsk, Russia \\ 3 Sobolev Institute of Geology and Mineralogy, Siberian Branch of the Russian Academy of Sciences, \\ 630090 Novosibirsk, Russia; victoria.saratovkina@gmail.com \\ * Correspondence: kev604@mail.ru
}

check for updates

Citation: Kaneva, E.; Radomskaya, T.; Shendrik, R.; Chubarov, V.; Danilovsky, V. Potassic-Hastingsite from the Kedrovy District (East Siberia, Russia): Petrographic Description, Crystal Chemistry, Spectroscopy, and Thermal Behavior. Minerals 2021, 11, 1049. https:// doi.org/10.3390/min11101049

Academic Editors: Gioacchino Tempesta and Giovanna Agrosì

Received: 21 August 2021

Accepted: 24 September 2021

Published: 27 September 2021

Publisher's Note: MDPI stays neutral with regard to jurisdictional claims in published maps and institutional affiliations.

Copyright: (c) 2021 by the authors. Licensee MDPI, Basel, Switzerland. This article is an open access article distributed under the terms and conditions of the Creative Commons Attribution (CC BY) license (https:/ / creativecommons.org/licenses/by/ $4.0 /)$.
Abstract: In this work we report on a petrographic, crystal-chemical, and optical characterization, obtained from different analytical methods, of amphibole species. Potassic-hastingsite, ideally ${ }^{\mathrm{A}} \mathrm{K}^{\mathrm{B}} \mathrm{Ca}_{2}{ }^{\mathrm{C}}\left(\mathrm{Fe}^{2+}{ }_{4} \mathrm{Fe}^{3+}\right)^{\mathrm{T}}\left(\mathrm{Si}_{6} \mathrm{Al}_{2}\right) \mathrm{O}_{22}{ }^{\mathrm{W}}(\mathrm{OH})_{2}$, has been found in the Kedrovy district (East Siberia, Russia). The sample occurs as well-formed and large radially radiant aggregates of dark green, almost black crystals. The unit cell dimensions are $a=9.9724(3) \AA, b=18.2968(4) \AA, c=5.3573(1)$ $\AA, \beta=104.945(3)^{\circ}, V=944.44(4) \AA^{3}, Z=2$. Site populations were determined by combining singlecrystal structure refinement and electron probe microanalysis, and $\mathrm{Fe}^{3+} / \mathrm{Fe}^{2+}$ ratio was obtained from X-ray fluorescence analysis. Infrared, diffuse light UV/Vis/NIR absorption, and electron spin resonance spectra are presented and discussed. A thermoelastic behavior of a powder of potassichastingsite was studied by in situ high-temperature X-ray diffraction. A thermal expansion and subsequent significant contraction in the unit cell volume during a high-temperature $\mathrm{X}$-ray powder diffraction experiment is observed as a consequence of the deprotonation process, which is locally balanced via oxidation of $\mathrm{Fe}^{2+}$. According to the data obtained for potassic-hastingsite, these processes occur within $400-600{ }^{\circ} \mathrm{C}$. The thermal expansion of the mineral is anisotropic; the thermal expansivity coefficients $\alpha_{a}: \alpha_{b}: \alpha_{c}\left(\times 10^{-6}\right)=-18.06: 9.59:-1.09$ at $400{ }^{\circ} \mathrm{C},-26.15:-1.52: 2.22$ at $600{ }^{\circ} \mathrm{C}$ and 23.77:-25.06:42.08 at $750{ }^{\circ} \mathrm{C}$.

Keywords: potassic-hastingsite; amphiboles; petrography; mineral associations; crystal chemistry; IR spectroscopy; electron spin resonance; absorption spectroscopy; thermal behavior

\section{Introduction}

Hastingsite is an end-member of the calcium amphiboles subgroup [1], which form translucent to opaque platy or grainy, acicular, radiating, fibrous, columnar crystals having a black, dark-green, greenish-brown, tan, or brown to yellow color. Hastingsite was discovered and named in 1896 by F. D. Adams and B. J. Harrington for the type locality in Hastings County (Canada).

According to the nomenclature of the amphibole-supergroup [2,3] the general formula of the amphiboles is $\mathrm{AB}_{2} \mathrm{C}_{5} \mathrm{~T}_{8} \mathrm{O}_{22} \mathrm{~W}_{2}$, where $\mathrm{A}=\square, \mathrm{Na}, \mathrm{K}, \mathrm{Ca}, \mathrm{Pb}, \mathrm{Li} ; \mathrm{B}=\mathrm{Na}, \mathrm{Ca}, \mathrm{Mn}^{2+}$, $\mathrm{Fe}^{2+}, \mathrm{Mg}, \mathrm{Li} ; \mathrm{C}=\mathrm{Mg}, \mathrm{Fe}^{2+}, \mathrm{Mn}^{2+}, \mathrm{Al}, \mathrm{Fe}^{3+}, \mathrm{Mn}^{3+}, \mathrm{Ti}^{4+}, \mathrm{Li} ; \mathrm{T}=\mathrm{Si}, \mathrm{Al}, \mathrm{Ti}^{4+}, \mathrm{Be} ; \mathrm{W}=(\mathrm{OH}), \mathrm{F}$, $\mathrm{Cl}, \mathrm{O}^{2-}$.

Hastingsite has the ideal formula $\mathrm{NaCa}_{2}\left(\mathrm{Fe}^{2+}{ }_{4} \mathrm{Fe}^{3+}\right)\left(\mathrm{Si}_{6} \mathrm{Al}_{2}\right) \mathrm{O}_{22}(\mathrm{OH})_{2}$. The observed variations in the end-member formula recognized by the IMA for hastingsite are reported in Table 1. Detailed crystal-chemical properties of the minerals have been investigated, sometimes using a multi-technique approach consisting of electron probe microanalysis, Mössbauer spectroscopy, and single-crystal X-ray refinements [4-21]. However, rela- 
tively few studies on their structural behavior under non-ambient conditions have been made $[22,23]$.

Table 1. Previously published selected data on hastingsites.

\begin{tabular}{|c|c|c|c|c|}
\hline $\begin{array}{c}\text { Name } \\
\text { (Year of Naming) }\end{array}$ & Chemical Formula & Type Locality & Ref. & Abbr. \\
\hline $\begin{array}{l}\text { Hastingsite } \\
\quad(1896)\end{array}$ & $\mathrm{NaCa}_{2}\left(\mathrm{Fe}^{2+}{ }_{4} \mathrm{Fe}^{3+}\right)\left(\mathrm{Si}_{6} \mathrm{Al}_{2}\right) \mathrm{O}_{22}(\mathrm{OH})_{2}$ & $\begin{array}{l}\text { Dungannon Township, } \\
\text { Hastings County, Ontario, } \\
\text { Canada. }\end{array}$ & [4-6] & Hs \\
\hline $\begin{array}{c}\text { Potassic-hastingsite } \\
\text { (2020) }\end{array}$ & $\mathrm{KCa}_{2}\left(\mathrm{Fe}^{2+}{ }_{4} \mathrm{Fe}^{3+}\right)\left(\mathrm{Si}_{6} \mathrm{Al}_{2}\right) \mathrm{O}_{22}(\mathrm{OH})_{2}$ & $\begin{array}{c}\text { Keshiketeng Banner, Inner } \\
\text { Mongolia, China }\end{array}$ & [7-10] & $\mathrm{K}-\mathrm{Hs}$ \\
\hline $\begin{array}{l}\text { Potassic-fluoro-hastingsite } \\
\text { (2009) }\end{array}$ & $\mathrm{KCa}_{2}\left(\mathrm{Fe}^{2+}{ }_{4} \mathrm{Fe}^{3+}\right)\left(\mathrm{Si}_{6} \mathrm{Al}_{2}\right) \mathrm{O}_{22} \mathrm{~F}_{2}$ & $\begin{array}{l}\text { Greenwood mine, Town of } \\
\text { Tuxedo, New York, USA }\end{array}$ & [11] & K-F-Hs \\
\hline $\begin{array}{l}\text { Potassic-chloro-hastingsite } \\
\qquad(2005)\end{array}$ & $\mathrm{KCa}_{2}\left(\mathrm{Fe}^{2+}{ }_{4} \mathrm{Fe}^{3+}\right)\left(\mathrm{Si}_{6} \mathrm{Al}_{2}\right) \mathrm{O}_{22} \mathrm{Cl}_{2}$ & $\begin{array}{c}\text { Dashkesan Co-Fe deposit, } \\
\text { Dashkesan district, } \\
\text { Azerbaijan }\end{array}$ & {$[12,13]$} & $\mathrm{K}-\mathrm{Cl}-\mathrm{Hs}$ \\
\hline $\begin{array}{c}\text { Magnesio-hastingsite } \\
(1928)\end{array}$ & $\mathrm{NaCa}_{2}\left(\mathrm{Mg}_{4} \mathrm{Fe}^{3+}\right)\left(\mathrm{Si}_{6} \mathrm{Al}_{2}\right) \mathrm{O}_{22}(\mathrm{OH})_{2}$ & $\begin{array}{l}\text { Mont Royal, Monreal, } \\
\text { Quebec, Canada }\end{array}$ & [14-16] & $\mathrm{Mg}-\mathrm{Hs}$ \\
\hline $\begin{array}{l}\text { Potassic-magnesio-hastingsite } \\
(2006)\end{array}$ & $\mathrm{KCa}_{2}\left(\mathrm{Mg}_{4} \mathrm{Fe}^{3+}\right)\left(\mathrm{Si}_{6} \mathrm{Al}_{2}\right) \mathrm{O}_{22}(\mathrm{OH})_{2}$ & $\begin{array}{l}\text { Ylmenogorsky complex, } \\
\text { South Urals, Russia }\end{array}$ & {$[17,18]$} & $\mathrm{K}-\mathrm{Mg}-\mathrm{Hs}$ \\
\hline $\begin{array}{c}\text { Magnesio-fluoro-hastingsite } \\
\text { (2006) }\end{array}$ & $\mathrm{NaCa}_{2}\left(\mathrm{Mg}_{4} \mathrm{Fe}^{3+}\right)\left(\mathrm{Si}_{6} \mathrm{Al}_{2}\right) \mathrm{O}_{22} \mathrm{~F}_{2}$ & $\begin{array}{l}\text { Uroi Hill, Simeria, } \\
\text { Hunedoara, Romania }\end{array}$ & {$[19,20]$} & $\mathrm{Mg}-\mathrm{F}-\mathrm{Hs}$ \\
\hline $\begin{array}{l}\text { Oxo-magnesio-hastingsite } \\
\text { (2013) }\end{array}$ & $\mathrm{NaCa}_{2}\left(\mathrm{Mg}_{2} \mathrm{Fe}^{3+}{ }_{3}\right)\left(\mathrm{Si}_{6} \mathrm{Al}_{2}\right) \mathrm{O}_{22} \mathrm{O}_{2}$ & $\begin{array}{c}\text { Deeti volcanic cone, } \\
\text { Gregory rif, northern } \\
\text { Tanzania }\end{array}$ & [21] & $\mathrm{O}-\mathrm{Mg}-\mathrm{Hs}$ \\
\hline
\end{tabular}

This work is devoted to the description of a new occurrence of potassic-hastingsite found in the Kedrovy district (East Siberia, Russia). Despite the fact that the mineral and the name were approved by IMA-CNMMN in 2018, and the description of the holotype was published in 2020 [10], this species of hastingsite was widely known and reported in early works. High-potassium hastingsites from Yukon (Canada) [7], West Ongul Island (East Antarctica) [8], and Vlastějovice (Czech Republic) [9] were described before approval.

In this paper we report the results of the petrographic study, crystal-chemical characterization, and thermal behavior investigation of potassic-hastingsite. Attention is focused on the thermal expansion and structural evolution with temperature. Additional infrared spectroscopy, diffuse-light absorption spectroscopy in the ultraviolet (UV), visible (Vis) and near infrared (NIR) spectral region, and electron spin resonance studies were carried out for a more detailed characterization of the optical properties of the studied potassic-hastingsite and the processes occurring during its heating.

\section{Materials and Methods}

\subsection{Sample Description}

A skarn sample containing potassic-hastingsite used for this study is derived from the collection of the Sidorov Mineralogical museum of INRTU (Irkutsk, Russia). The specimen was found in the Kedrovy district, located $10 \mathrm{~km}$ northeast of the urban Vitimskoye settlement upstream of the Malaya Severnaya River (Mamsko-Chuisky area, Irkutsk Region, East Siberia, Russia) (Figure 1). The skarn sample was taken in the zone of contact-metasomatic impact of Paleozoic granitoids (Konkudero-Mamakan and Mamsk complexes) on the host strata (marbleized limestones and shales of the Sogdiondonskay Formation $\left(\mathrm{PR}_{3} \mathrm{sg}\right)$ ).

The morphogenetic features of potassic-hastingsite and its paragenetic relationship with associated minerals were studied by optical petrographic methods using an Olympus BX-51 polarizing microscope (Olympus Co., Tokyo, Japan) in transmitted light.

The sample is a rock fragment $(7 \mathrm{~cm} \times 4 \mathrm{~cm} \times 3 \mathrm{~cm})$, composed mainly of intergrowths and radial-radiant aggregates of dark green, almost black potassic-hastingsite, brownorange garnet, purple vesuvianite, and small amounts of calcite, quartz, and sulfides 
(Figure 2). Apatite and zircon were identified as accessory minerals. Zoisite and epidote are found as single grains. Minerals are unevenly distributed. The structure is coarse-grained.

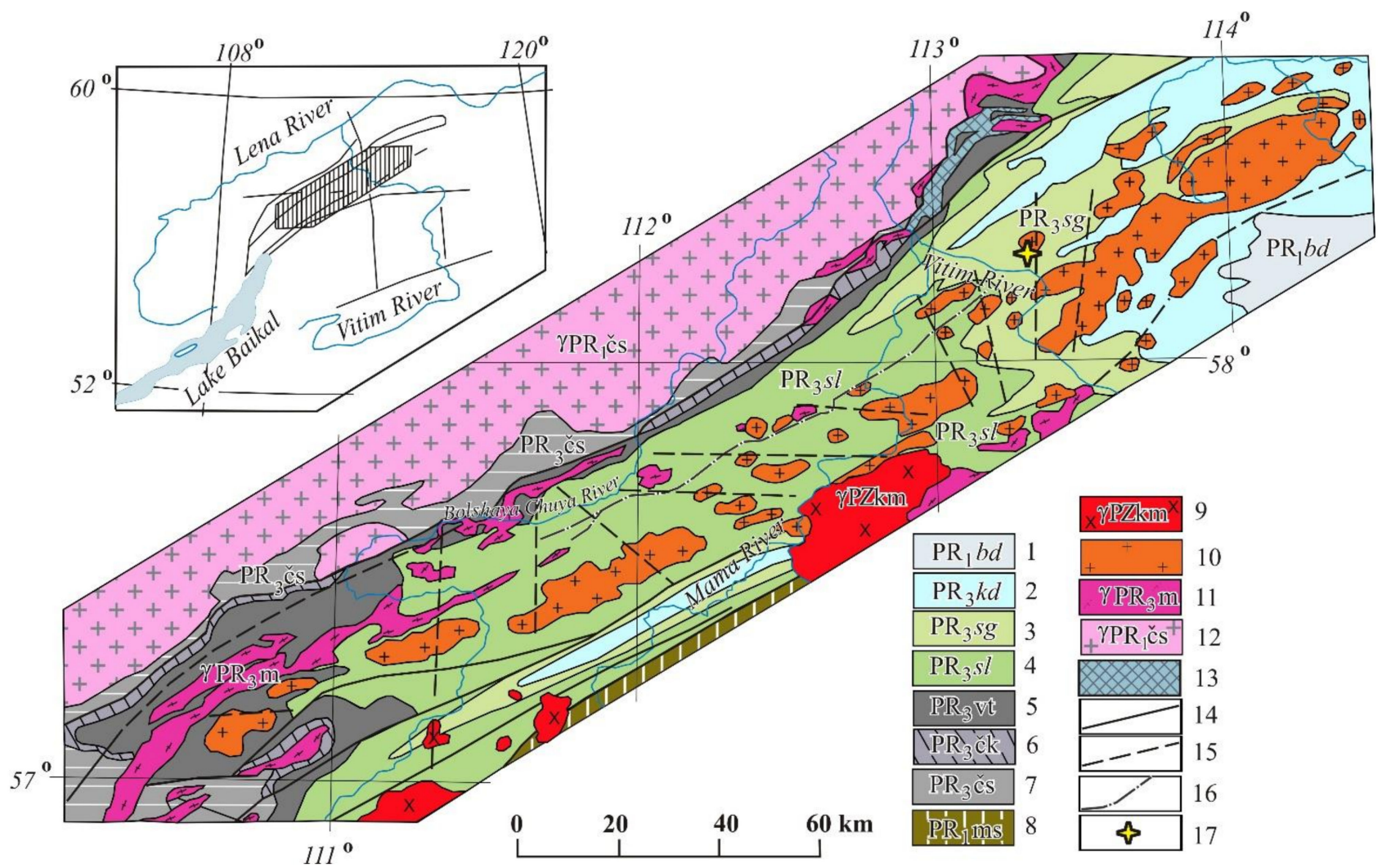

Figure 1. Geological map of the Mamsksk mica-bearing province modified after [24]. 1-Bodaibo subseries: Vachskaya, Anangrskaya, Dogaldynskaya and Iligirskaya combined formations-carbonaceous quartzites, carbonaceous shales, sandstones and siltstones, phyllitic shales; 2-Kadalikanskaya subseries: Konkuderskaya, Shusmanskaya, Khomolkhinskaya, Imnyakhskaya, Bodaibokanskaya combined formations-scapolite-biotite and biotite spotted shales and crystalline limestones, marbles, in zones of low-grade metamorphism—sericite-quartz "carbonaceous" limestones; 3-6- Mamskaya subseries: 3-Sogdondonskaya suite, 4-Slyudyankinskaya suite, 5-Vitimskaya suite, 6-Chukchinskaya and Yakdakarskaya combined formations-metagravelites and metaarkosic sandstones, quartzites, amphibolites and amphibole gneisses, disten-garnet-staurolite and two-mica schists; 7-Chuyskaya and Ukuchiktinskaya strata - migmatites and granite-gneisses, biotite, hornblende-biotite and pyroxene gneisses, sillimanite schists; 8-Muyskaya strata—sandstones, siltstones, chloritebiotite, quartz-sericite and biotite schists, quartzites, orthoschists; 9-Konkudero-Mamakan complex-amphibole-biotite and biotite granites, granodiorites and granosyenites; 10-11-Mamsko-Oron complex: 10—Second phase: granite-pegmatites, K-feldspar pegmatites, mica-bearing pegmatites; 11-first phase: amphibole-biotite and biotite gneiss-granites, plagiogranites, basic plagioclase pegmatites; 12 - Chuya-Kodar complex-porphyritic and uniformly granular hornblende-biotite and biotite granites, leucocratic granites, plagiogranites, pegmatites; 13-orthoamphibolites; 14—tectonic faults tracked and confirmed by geophysical data; 15-alleged tectonic faults based on geophysical data, 16-thrust zones filled with tectonites, migmatized gneisses with magnetite; 17—sampling point.

In the thin section, euhedral and subhedral elongated prismatic crystals of amphibole $(10 \mathrm{~mm} \times 2.5 \mathrm{~mm} \times 1.5 \mathrm{~mm})$ exhibit pleochroism, which is characteristic of the hastingsites: light brownish green on $\mathrm{Np}$, dark olive-green on $\mathrm{Nm}$, blue-green on $\mathrm{Ng}$ (Figure 3a,b). Potassic-hastingsite crystals are anhedral relatively to euhedral garnet crystals (Figure 3c,d). Amphibole contains inclusions of colorless euhedral prismatic elongated crystals of apatite (up to $0.5 \mathrm{~mm} \times 0.3 \mathrm{~mm}$ in size) (Figure 3e,f) and zircon grains (up to $0.2 \mathrm{~mm} \times 0.1 \mathrm{~mm}$ in size). Around zircon enclosed in amphibole, pleochroic haloes are formed. Zircon is most likely a relict mineral. Sulfides (pyrite) and the products of their oxidation (minerals of the limonite group) (Figure $3 \mathrm{~g}, \mathrm{~h}$ ) are found along cleavage and cataclase cracks in potassic-hastingsite. 


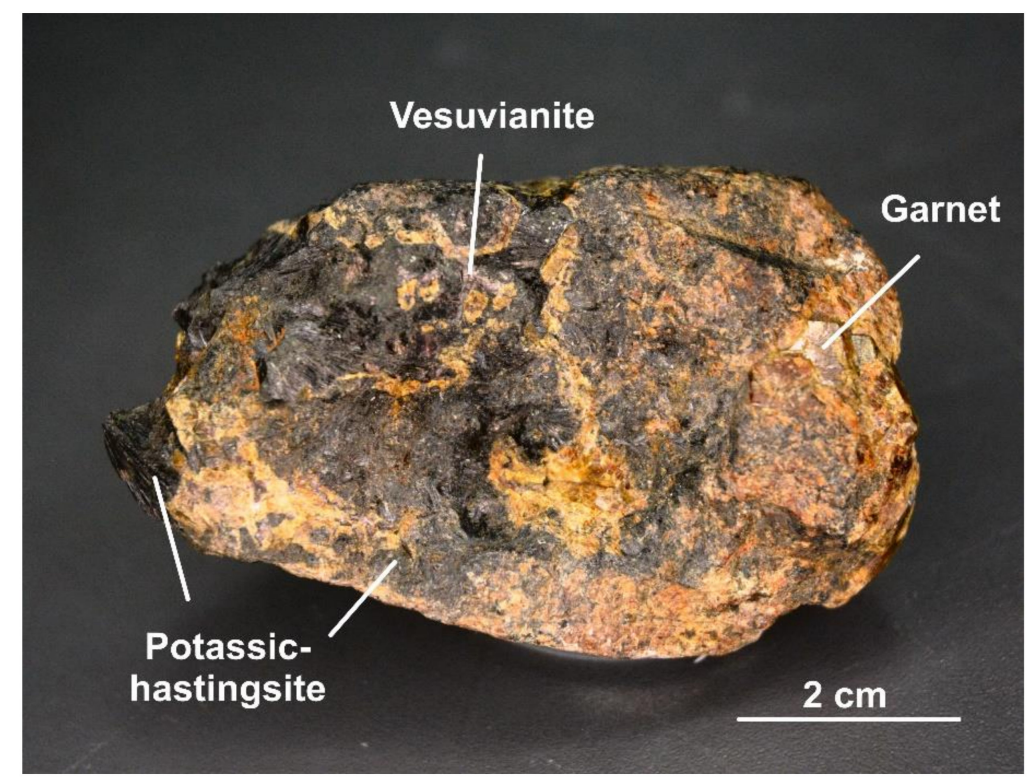

Figure 2. Skarn sample.
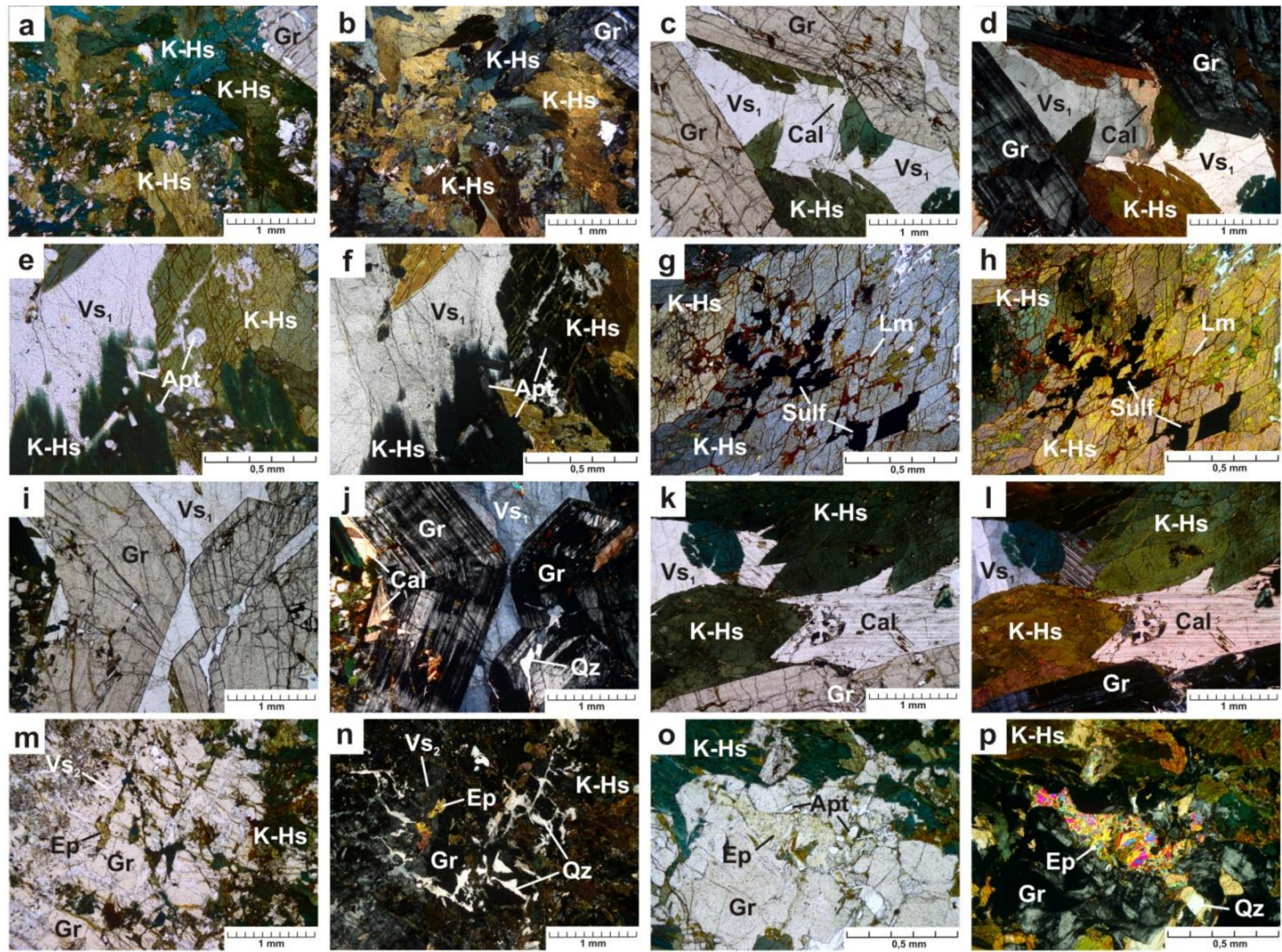

Figure 3. Photomicrographs of the mineral assemblages and their textural properties in a thin section in transmitted light, $(\mathbf{a}, \mathbf{c}, \mathbf{e}, \mathbf{g}, \mathbf{i}, \mathbf{k}, \mathbf{m}, \mathbf{o})$ - plane polarised light and $(\mathbf{b}, \mathbf{d}, \mathbf{f}, \mathbf{h}, \mathbf{j}, \mathbf{l}, \mathbf{n}, \mathbf{p})$-cross polarised light image. Cal—calcite, Ep-epidote, Gr-garnet, K-Hs—potassic-hastingsite, Lm-minerals of the limonite group, Qz-quartz, Sulf-sulfides, Vs $\mathrm{s}_{1}$ and $\mathrm{Vs}_{2}$ - vesuvianite of the first and second generation, respectively. Mineral abbreviations are used according to [3,25]. 
Garnet crystals in the thin section are skeletal formations up to $1 \mathrm{~cm}$ in size with a faint yellow tint. The central parts of them are filled with intergrowths of garnet, potassic-hastingsite, and vesuvianite, while the peripheral part is composed only of garnet (Figure 3i,j). Moreover, the edges are euhedral. Through crossed polarizers, the mineral exhibits anomalous interference coloration: alternating isotropic and anisotropic zones (Figure 3j). Such zoning is typical for grossular-andradite garnets [26-28]. Garnet grains are broken by numerous cataclase cracks filled with vesuvianite, quartz, and sulfides.

Colorless subhedral crystals of calcite (up to $2 \mathrm{~mm}$ ) are irregularly distributed and anhedral relative to potassic-hastingsite and garnet (Figure $3 \mathrm{c}, \mathrm{d}, \mathrm{k}, \mathrm{l}$ ).

Vesuvianite is found in two generations. The first one is associated with potassichastingsite, garnet, and calcite, in relation to which the mineral is anhedral (Figure 3c-f,i-1). The grain size varies from 1 to $4 \mathrm{~mm}$. Vesuvianite of the second generation fills the cracks of cataclase (Figure $3 \mathrm{~m}, \mathrm{n})$. Its grains exhibit weak pleochroism, from light lilac to light yellow, and anomalous interference coloration of brownish lilac and gray.

Quartz fills the cracks of cataclase, and has a superimposed nature of formation (Figure $3 \mathrm{~m}-\mathrm{p}$ ). Large sulfide grains (up to $1 \mathrm{~mm}$ ) are euhedral and subhedral. They have a characteristic rim of iron hydroxides. Sulfides were also found filling cleavage and catclase cracks.

In thin sections, single grains of epidote and zoisite can be noted (Figure $3 \mathrm{~m}-\mathrm{p}$ ).

\subsection{Chemical Analysis}

Mineral chemistry was analyzed in $\sim 0.2-0.6 \mathrm{~mm}$ grains on a JEOL JXA-8230 electron probe microanalyzer (Jeol, Tokyo, Japan). The electron probe microanalyses (EPMA) were performed with a $20 \mathrm{kV}$ accelerating voltage, $10 \mathrm{nA}$ beam current, and a $\sim 10 \mu \mathrm{m}$ spot size in wavelength-dispersive spectroscopic (WDS) mode. The composition was determined with reference to the following standards: $\mathrm{F}$ - phlogopite ( $\mathrm{Si}, \mathrm{Al}, \mathrm{K}, \mathrm{Mg}, \mathrm{F}$ ), diopside (Ca), albite (Na), Mn-garnet rhodonite $(\mathrm{Mn})$, pyrope (Fe), Ti-glass (Ti), $\mathrm{Cl}$-apatite $(\mathrm{Cl})$, and $\mathrm{Cr}$-pyrope $(\mathrm{Cr})$. A conversion from X-ray counts to oxide weight percentages (wt\%) was obtained with the ZAF matrix correction [29]. Chemical composition results, obtained from 16 analyses, show that the samples examined are relatively homogeneous: $\mathrm{Al}_{2} \mathrm{O}_{3}(10.4-11.2 \mathrm{wt} \%), \mathrm{SiO}_{2}(36.2-37.1 \mathrm{wt} \%), \mathrm{Na}_{2} \mathrm{O}(0.8-1.2 \mathrm{wt} \%), \mathrm{CaO}(10.9-11.2 \mathrm{wt} \%)$, $\mathrm{K}_{2} \mathrm{O}(2.0-2.3 w \mathrm{t} \%), \mathrm{FeO}(33.2-34.3 \mathrm{wt} \%), \mathrm{MgO}(0.8-1.2 \mathrm{wt} \%), \mathrm{MnO}(0.34-0.40 \mathrm{wt} \%), \mathrm{TiO}_{2}$ $(0.20-0.24 \mathrm{wt} \%), \mathrm{Cl}(1.8-2.3 \mathrm{wt} \%)$, and $\mathrm{F}(0.11-0.20 \mathrm{wt} \%)$ contents (97.9-98.4 wt $\%$ total). $\mathrm{OH}$-group content was calculated from the charge balance.

Determination of $\mathrm{Fe}^{2+}$ and $\mathrm{Fe}^{3+}$ was performed using the X-ray fluorescence (XRF) technique based on the measurement of the relative intensity of the $\mathrm{X}$-ray emission line influenced by the iron chemical state $\left(\mathrm{FeK} \beta_{5}\right)$. This technique was developed for rocks analysis [30-32] and successfully applied for iron valence state assessment in picroilmenites [33]. Calibration curves were constructed using certified reference materials of igneous rock of ultrabasic composition with certified values of total and ferrous iron, iron minerals (hematite, magnetite), and amphibole samples analyzed by certified volumetric and atomic absorption techniques. Samples were prepared as pressed pellets on a boric acid substrate. Measurements were performed on a wavelength-dispersive X-ray fluorescence spectrometer S4 Pioneer (Bruker AXS, Berlin, Germany).

\subsection{Structural Analysis}

Single crystal X-ray diffraction analysis (SCXRD) of the selected single crystal was carried out by means of a Bruker AXS D8 VENTURE automated diffractometer (Bruker AXS, Berlin, Germany) with graphite-monochromatized MoK $\alpha$ radiation at room temperature. Operating conditions were: $50 \mathrm{kV}$ and $1 \mathrm{~mA}$, with a crystal-to-detector distance of $40 \mathrm{~mm}$. A set of 24 frames was used for initial cell determination with a strategy optimized by the APEX2 suite package [34]. Data collection was accomplished by several $\varphi$ and $\omega$ scans with $0.3^{\circ}$ rotation and $4.3 \mathrm{~s}$ exposure time per frame. The half of the Ewald sphere $( \pm h$, $\pm k, \pm l$ ) was recorded up to $\theta_{\max } \sim 40^{\circ} .40,291$ reflections were measured, of which 2568 
were independent. Data reduction was performed using CrysAlisPro Version 1.171.39.46 (Rigaku, Tokyo, Japan) [35]. Anisotropic structure refinement was carried out using the program CRYSTALS [36] in the space group $C 2 / m$. The refined parameters were: scale factor, atom positions, anisotropic displacement parameters. In the refinement, the site population and nomenclature defined by $[37,38]$ were used. Unit cell parameters, some details on data collection, and summary results from structure refinements are reported in Table 2.

Table 2. Selected data on single crystal, data collection, and structure refinement parameters of the studied potassic-hastingsite sample.

\begin{tabular}{|c|c|c|c|}
\hline \multicolumn{2}{|c|}{ Crystal Data } & \multicolumn{2}{|c|}{ Data Collection } \\
\hline Crystal system & Monoclinic & Theta range & $2.226^{\circ}$ to $38.221^{\circ}$ \\
\hline Space group & $\mathrm{C} 2 / \mathrm{m}$ & Reflection measured & 40,291 \\
\hline Temperature $(\mathrm{K})$ & 293 & Independent reflections & 2568 \\
\hline$a(\AA)$ & $9.9724(3)$ & $R_{\text {merging }}\left[R_{(\mathrm{int})}\right](\%)$ & 3.70 \\
\hline$b(\AA)$ & $18.2968(4)$ & $h_{\min }, h_{\max }$ & $-17,17$ \\
\hline$c(\AA)$ & $5.3573(1)$ & $k_{\min }, k_{\max }$ & $-31,31$ \\
\hline$\beta\left(^{\circ}\right)$ & 104.945(3) & $l_{\min }, l_{\max }$ & $-9,9$ \\
\hline$V\left(\AA^{3}\right)$ & $944.44(4)$ & \multicolumn{2}{|c|}{ Refinement } \\
\hline Z & 2 & $\begin{array}{l}\text { Reflections used in the } \\
\text { refinement }\left(\mathrm{I}>3 \sigma_{(\mathrm{I})}\right)\end{array}$ & 1851 \\
\hline Radiation type & X-ray, $\lambda=0.7107 \AA$ & N. of refined parameters & 124 \\
\hline Crystal shape & tabular & $R^{\mathrm{a}}[$ on $F](\%)$ & 2.71 \\
\hline Crystal dimensions (mm) & $0.14 \times 0.10 \times 0.08$ & $R_{\mathrm{W}}{ }^{\mathrm{b}}[\mathrm{on} F](\%)$ & 2.92 \\
\hline Crystal color & brownish-black & Goof $^{c}$ & 1.0726 \\
\hline Calculated density $\left(\mathrm{g} / \mathrm{cm}^{3}\right)$ & 3.485 & $\Delta \rho_{\min } / \Delta \rho_{\max }\left(\mathrm{e}^{-} / \AA^{3}\right)$ & $-0.79 / 0.88$ \\
\hline
\end{tabular}

${ }^{\mathrm{a}} R=\Sigma\left[\left|F_{\mathrm{o}}\right|-\left|F_{\mathrm{c}}\right|\right] / \Sigma\left|F_{\mathrm{o}}\right| .{ }^{\mathrm{b}} R_{\mathrm{w}}=\left[\Sigma\left[w\left(F_{\mathrm{o}}{ }^{2}-{F_{c}}^{2}\right)^{2}\right] / \Sigma\left[w\left(F_{\mathrm{o}}{ }^{2}\right)^{2}\right]\right]^{1 / 2} ; w=$ Chebyshev optimized weights. ${ }^{c}$ Goodness-of-fit $=\left[\Sigma\left[w\left(F_{o}^{2}-F_{c}^{2}\right)^{2}\right] /(N-p]^{1 / 2}\right.$, where $\mathrm{N}$ and $\mathrm{p}$ are the number of reflections and parameters, respectively. $F-$ structure factor.

The CIF was deposited with the Cambridge Crystallographic Data Centre (CSD 2102019).

A figure showing structural details was prepared using the program VESTA (version 4.3.0, Tsukuba, Japan) [39].

High-temperature X-ray powder diffraction (HTXRPD) data of the studied sample were collected in air with a Bruker D8 ADVANCE powder diffractometer (Bruker AXS, Berlin, Germany) with Bragg-Brentano geometry (Cu-K $\alpha$ radiation, $40 \mathrm{kV}, 40 \mathrm{Ma})$. The XRPD system is equipped with a HTK16 heating chamber. The powder was deposited on a $\mathrm{PtRh}$ substrate, assuring a direct homogeneous heating and fast temperature ramping. The sample was found to be single phase.

Profiles were obtained between $7^{\circ}$ and $65^{\circ} 2 \theta$ with a step size of $2 \theta-0.02^{\circ}$ and a counting time of $1 \mathrm{~s}$ per step. The temperature step of the heating and cooling rate was $25^{\circ} \mathrm{C} /$ min stepwise at discrete steps of $50^{\circ} \mathrm{C}$ up to $750{ }^{\circ} \mathrm{C}$ and of $100{ }^{\circ} \mathrm{C}$ down to $30^{\circ} \mathrm{C}$ with an equilibration time of $2 \mathrm{~min}$.

The structural model of potassic-hastingsite obtained by SCXRD was taken as a starting for the refinement of the unit cell parameters performed using TOPAS 4.2 software package (Bruker AXS, Berlin, Germany) [40]. The background was modeled using a Chebychev polynomial approximation of 6th order. The peak profile was described by a pseudo-Voight. The coefficients of thermal expansion tensor and the orientation of the principal axes of the thermal-expansion tensor with respect to the crystallographic axes were determined using the TEV program [41].

\subsection{Spectroscopy Study}

In order to obtain infrared (IR) absorption spectra of potassic-hastingsite, powdered samples were mixed with anhydrous $\mathrm{KBr}$, pelletized, and analyzed using an FT-801 spectrometer (Simex, Novosibirsk, Russia) at a resolution of $2 \mathrm{~cm}^{-1}$. A total of 32 scans were collected for each spectrum. The IR spectrum of an analogous pellet of pure $\mathrm{KBr}$ was used as a reference. 
Diffuse-light absorption spectra in the ultraviolet (UV), visible (Vis) and near infrared (NIR) spectral region were recorded at room temperature with a PerkinElmer Lambda 950 spectrophotometer (Perkin-Elmer, Shelton, CT, USA) in an integrating sphere. For measurements, the powder samples of potassic-hastingsite were placed in a quartz test tube, which is transparent in the range of 250-2000 nm.

Electron spin resonance (ESR) spectra were registered using a RE-1306 X-band spectrometer (KBST, Smolensk, Russia) with a frequency of $9.310 \mathrm{GHz}$. Powder of potassichastingsite was placed in a quartz test tube. The measurements were carried out at room temperature.

\section{Results}

\subsection{Petrographic Analysis}

The investigation of the morphogenetic features of potassic-hastingsite and its paragenetic associations allowed us to determine the sequence of the mineral crystallization of the studied skarn sample (Table 3).

Table 3. Schematic diagram showing paragenetic relationships of mineral assemblages in the skarn sample, based on paragenetic relation and microtextural data.

\begin{tabular}{lll}
\hline \multicolumn{1}{c}{ Mineral } & \multicolumn{2}{c}{ Skarn Formation Stages } \\
\cline { 2 - 3 } Garnet (grossular-andradite) & Late Hydrosilicatic Stage \\
Apatite & \\
Amphibole \\
(potassic-hastingsite)
\end{tabular}

\subsection{Chemical Composition and Structure Description}

Combined EPMA (determined over 16 spots) and XRF results obtained for the studied potassic-hastingsite sample are reported in Table 4, compared with those in the literature for the hastingsites. The results of the XRF show that the $\mathrm{Fe}^{2+}$ is the principal oxidation state in the mineral $\left(\mathrm{Fe}^{2+}=74 \%\right)$. The empirical formula was calculated based on fixing the sum of $\mathrm{O}+\mathrm{OH}+\mathrm{Cl}+\mathrm{F}$ to 24 by means of the amphibole classification spreadsheet (August 2020 version) originally published by [42]. The formula is represented in Table 5, where the information on the literature crystal chemistry of hastingsites is also summarized. 


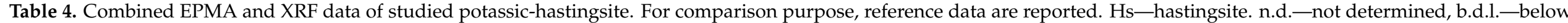
detection limit.

\begin{tabular}{|c|c|c|c|c|c|c|c|c|c|c|c|c|c|}
\hline Constituent & $\begin{array}{c}\text { K-Hs, } \\
\text { Kedrovy } \\
\text { District, } \\
\text { Russia } \\
\text { This study }\end{array}$ & $\begin{array}{c}\text { K-Hs, Yukon, } \\
\text { Canada } \\
{[7]}\end{array}$ & $\begin{array}{c}\text { K-Hs, } \\
\text { Keshiketeng } \\
\text { Banner, } \\
\text { China } \\
{[10]} \\
\end{array}$ & $\begin{array}{c}\text { K-Hs, } \\
\text { Vlastějovice, } \\
\text { Czech } \\
\text { Republic [9] }\end{array}$ & $\begin{array}{c}\text { K-Hs, West } \\
\text { Ongul } \\
\text { Island, East } \\
\text { Antarctica [8] }\end{array}$ & $\begin{array}{c}\text { K-Cl-Hs, } \\
\text { Dashkesan, } \\
\text { Azerbaidjan } \\
\text { [13] }\end{array}$ & $\begin{array}{c}\text { K-F-Hs, } \\
\text { Greenwood } \\
\text { mine, USA } \\
\text { [11] }\end{array}$ & $\begin{array}{c}\text { Mg-Hs, } \\
\text { Lafarge } \\
\text { Quarry, } \\
\text { Canada [16] }\end{array}$ & $\begin{array}{c}\text { Mg-F-Hs, } \\
\text { Eifel, } \\
\text { Rheinland-Pfalz, } \\
\text { Germany } \\
\text { [20] } \\
\end{array}$ & $\begin{array}{c}\text { Mg-F-Hs, } \\
\text { Uroi Hill, } \\
\text { Romania [19] }\end{array}$ & $\begin{array}{c}\text { K-Mg-Hs, } \\
\text { Ylmenogorsky } \\
\text { Complex, } \\
\text { South Urals, } \\
\text { Russia [18] }\end{array}$ & $\begin{array}{c}\text { K-Mg-Hs, } \\
\text { Prince Olav } \\
\text { Coast, East } \\
\text { Antarctica } \\
{[17]} \\
\end{array}$ & $\begin{array}{c}\text { O-Mg-Hs, } \\
\text { Deeti Volcanic } \\
\text { Cone, } \\
\text { Northern } \\
\text { Tanzania [21] }\end{array}$ \\
\hline $\mathrm{SiO}_{2}$ & $36.62(63)$ & $37.36(46)$ & 35.02 & 37.32 & 35.52 & 35.62 & 40.49 & 39.53 & 40.02 & 40.77 & 36.28 & 39.43 & 41.89 \\
\hline $\mathrm{Al}_{2} \mathrm{O}_{3}$ & $10.64(8)$ & $11.60(30)$ & 10.69 & 12.70 & 11.94 & 11.13 & 10.29 & 14.61 & 11.81 & 13.11 & 16.37 & 14.31 & 10.75 \\
\hline $\mathrm{Na}_{2} \mathrm{O}$ & $0.96(9)$ & $1.27(20)$ & 0.06 & 1.07 & 0.80 & 1.07 & 1.24 & 1.99 & 2.45 & 1.79 & 1.05 & 0.96 & 2.84 \\
\hline $\mathrm{MgO}$ & $0.96(8)$ & $0.98(26)$ & 0.23 & 3.83 & 4.44 & 2.91 & 6.68 & 14.07 & 15.24 & 18.70 & 10.23 & 9.02 & 14.79 \\
\hline $\mathrm{K}_{2} \mathrm{O}$ & $2.21(10)$ & $2.15(14)$ & 2.79 & 3.05 & 3.27 & 3.04 & 2.93 & 2.25 & 1.60 & 1.17 & 3.34 & 3.07 & 1.74 \\
\hline $\mathrm{CaO}$ & $11.05(9)$ & $11.28(22)$ & 11.35 & 11.38 & 11.32 & 10.72 & 11.13 & 11.89 & 12.28 & 13.99 & 11.84 & 12.23 & 11.76 \\
\hline $\mathrm{TiO}_{2}$ & $0.20(8)$ & $0.29(9)$ & 0.35 & 0.43 & 0.77 & 0.43 & 0.11 & 3.64 & 5.38 & 1.21 & 1.47 & 1.03 & 3.96 \\
\hline $\mathrm{V} 2_{3}$ & n.d. & n.d. & n.d. & n.d. & n.d. & n.d. & 0.03 & n.d. & b.d.l. & n.d. & b.d.l. & n.d. & trace \\
\hline $\mathrm{MnO}$ & $0.37(6)$ & $0.37(12)$ & 0.43 & 0.18 & 0.30 & 0.40 & 0.20 & 0.09 & b.d.l. & n.d. & 0.45 & 0.45 & 0.08 \\
\hline $\mathrm{FeO}$ & $27.66(52)$ & $25.70(2)$ & 26.08 & 20.81 & 19.73 & 23.48 & 19.80 & 5.45 & n.d. & n.d. & 8.76 & 8.38 & n.d. \\
\hline $\mathrm{Fe}_{2} \mathrm{O}_{3}$ & $6.92(51)$ & $6.55(2)$ & 7.33 & 6.64 & 7.44 & 7.80 & 4.49 & 4.76 & 9.16 & 6.44 & 7.29 & 9.88 & 11.25 \\
\hline $\mathrm{BaO}$ & n.d. & n.d. & n.d. & n.d. & n.d. & n.d. & n.d. & n.d. & b.d.l. & n.d. & 0.92 & n.d. & n.d. \\
\hline $\mathrm{F}$ & $0.17(5)$ & n.d. & 0.00 & 0.35 & 0.19 & 0.14 & 2.23 & 0.09 & 2.36 & 4.39 & n.d. & 0.20 & n.d. \\
\hline $\mathrm{Cl}$ & $2.08(17)$ & 2.35(39) & 2.79 & 0.24 & 3.27 & 4.68 & 0.61 & 0.02 & b.d.l. & n.d. & 0.5 & 0.10 & n.d. \\
\hline Sum & 99.84 & 99.98 & 97.15 & 98.00 & 98.99 & 101.42 & 100.24 & 98.42 & 100.3 & 101.57 & 98.50 & 99.08 & 99.06 \\
\hline $\mathrm{O}=\mathrm{F} 2$ & 0.07 & & & 0.15 & & & & & -0.99 & 1.84 & & & - \\
\hline $\mathrm{O}=\mathrm{Cl}$ & 0.47 & 0.53 & 0.67 & 0.05 & 0.82 & 1.12 & 1.08 & 0.04 & - & - & 0.1 & 0.11 & - \\
\hline Total & 99.30 & 99.45 & 96.48 & 97.80 & 98.17 & 100.30 & 99.16 & 98.38 & 99.31 & 99.73 & 98.40 & 98.97 & 99.06 \\
\hline
\end{tabular}

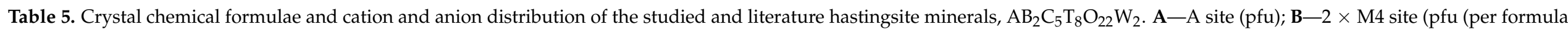
unit)); C-2 $\times$ M1, $2 \times$ M2, $1 \times$ M3 sites (pfu); T $-4 \times \mathrm{T} 1,4 \times \mathrm{T} 2$ sites (pfu); $\mathbf{W}-2 \times \mathrm{O} 3$ sites (pfu). The abbreviations of hastingsite species used are the same as in Table 1 .

\begin{tabular}{|c|c|c|c|c|c|c|}
\hline Mineral & A & B & C & $\mathrm{T}$ & $\mathbf{O}$ & W \\
\hline K-Hs, Kevrovy district, Russia This study & $\mathrm{K}_{0.46} \mathrm{Na}_{0.29}$ & $\mathrm{Ca}_{1.95} \mathrm{Na}_{0.03} \mathrm{Mn}_{0.02}$ & $\mathrm{Fe}^{2+}{ }_{3.49} \mathrm{Fe}^{3+}{ }_{1.15} \mathrm{Mg}_{0.24} \mathrm{Al}_{0.06} \mathrm{Mn}_{0.04} \mathrm{Ti}_{0.02}$ & $\mathrm{Si}_{6.01} \mathrm{Al}_{1.99}$ & $\mathrm{O}_{22}$ & $(\mathrm{OH})_{1.33} \mathrm{Cl}_{0.58} \mathrm{~F}_{0.09}$ \\
\hline K-Hs, Yukon, Canada [7] & $\mathrm{K}_{0.45} \mathrm{Na}_{0.40}$ & $\mathrm{Ca}_{1.97} \mathrm{Na}_{0.03}$ & $\mathrm{Fe}^{2+}{ }_{3.51} \mathrm{Fe}^{3+}{ }_{0.81} \mathrm{Al}_{0.32} \mathrm{Mg}_{0.24} \mathrm{Mn}_{0.05} \mathrm{Ti}_{0.03} \mathrm{Cr}_{0.01}$ & $\mathrm{Si}_{6.09} \mathrm{Al}_{1.91}$ & $\mathrm{O}_{22}$ & $(\mathrm{OH})_{1.38} \mathrm{Cl}_{0.62}$ \\
\hline K-Hs, Keshiketeng Banner, China [10] & $\mathrm{K}_{0.61} \mathrm{Na}_{0.20} \mathrm{Ca}_{0.07}$ & $\mathrm{Ca}_{2.00}$ & $\mathrm{Fe}^{2+}{ }_{3.72} \mathrm{Fe}^{3+}{ }_{0.94} \mathrm{Al}_{0.12} \mathrm{Mg}_{0.06} \mathrm{Mn}_{0.06} \mathrm{Ti}_{0.04} \mathrm{Ni}_{0.01}$ & $\mathrm{Si}_{5.97} \mathrm{Al}_{2.03}$ & $\mathrm{O}_{22}$ & $(\mathrm{OH})_{1.14} \mathrm{Cl}_{0.86}$ \\
\hline K-Hs, Vlastějovice, Czech Republic [9] & $\mathrm{K}_{0.62} \mathrm{Na}_{0.28}$ & $\mathrm{Ca}_{1.95} \mathrm{Na}_{0.05}$ & $\mathrm{Fe}^{2+}{ }_{2.79} \mathrm{Mg}_{0.92} \mathrm{Fe}^{3+}{ }_{0.80} \mathrm{Al}_{0.38} \mathrm{Ti}_{0.05} \mathrm{Mn}_{0.03}$ & $\mathrm{Si}_{5.98} \mathrm{Al}_{2.02}$ & $\mathrm{O}_{22}$ & $(\mathrm{OH})_{1.71} \mathrm{~F}_{0.18} \mathrm{Cl}_{0.06}$ \\
\hline K-Hs, West Ongul Island, East Antarctica [8] & $\mathrm{K}_{0.69} \mathrm{Na}_{0.26}$ & $\mathrm{Ca}_{1.99}$ & $\mathrm{Fe}^{2+}{ }_{2.71} \mathrm{Mg}_{1.09} \mathrm{Fe}^{3+}{ }_{0.92} \mathrm{Al}_{0.15} \mathrm{Ti}_{0.10} \mathrm{Mn}_{0.04}$ & $\mathrm{Si}_{5.84} \mathrm{Al}_{2.16}$ & $\mathrm{O}_{22}$ & $(\mathrm{OH})_{0.95} \mathrm{Cl}_{0.91} \mathrm{~F}_{0.10} \mathrm{O}_{0.04}$ \\
\hline K-Cl-Hs, Dashkesan, Azerbaidjan [13] & $\mathrm{K}_{0.64} \mathrm{Na}_{0.34}$ & $\mathrm{Ca}_{1.90}$ & $\mathrm{Fe}^{2+}{ }_{3.25} \mathrm{Fe}^{3+}{ }_{0.97} \mathrm{Mg}_{0.72} \mathrm{Mn}_{0.06} \mathrm{Al}_{0.06} \mathrm{Ti}_{0.05}$ & $\mathrm{Si}_{5.89} \mathrm{Al}_{2.11}$ & $\mathrm{O}_{22}$ & $\mathrm{Cl}_{1.31}(\mathrm{OH})_{0.60} \mathrm{~F}_{0.07} \mathrm{O}_{0.02}$ \\
\hline K-F-Hs, Greenwood mine, USA [11] & $\mathrm{K}_{0.59} \mathrm{Na}_{0.25}$ & $\mathrm{Ca}_{1.87} \mathrm{Na}_{0.13}$ & $\mathrm{Fe}^{2+}{ }_{2.60} \mathrm{Mg}_{1.56} \mathrm{Fe}^{3+}{ }_{0.53} \mathrm{Al}_{0.26} \mathrm{Mn}_{0.03} \mathrm{Ti}_{0.01}$ & $\mathrm{Si}_{6.36} \mathrm{Al}_{1.64}$ & $\mathrm{O}_{22}$ & $\mathrm{~F}_{1.11}(\mathrm{OH})_{0.73} \mathrm{Cl}_{0.16}$ \\
\hline Mg-Hs, Lafarge Quarry, Canada [16] & $\mathrm{Na}_{0.57} \mathrm{~K}_{0.42} \mathrm{Ca}_{0.01}$ & $\mathrm{Ca}_{1.88} \mathrm{Fe}^{2+}{ }_{0.11} \mathrm{Na}_{0.02} \mathrm{Mn}_{0.01}$ & $\mathrm{Mg}_{3.10} \mathrm{Fe}^{2+}{ }_{0.57} \mathrm{Fe}^{3+}{ }_{0.53} \mathrm{Ti}_{0.41} \mathrm{Al}_{0.39} \mathrm{Mn}_{0.02}$ & $\mathrm{Si}_{5.85} \mathrm{Al}_{2.15}$ & $\mathrm{O}_{22}$ & $(\mathrm{OH})_{1.36} \mathrm{O}_{0.59} \mathrm{~F}_{0.04} \mathrm{Cl}_{0.01}$ \\
\hline Mg-F-Hs, Eifel, Rheinland-Pfalz, Germany [20] & $\mathrm{Na}_{0.65} \mathrm{~K}_{0.30}$ & $\mathrm{Ca}_{1.95} \mathrm{Na}_{0.05}$ & $\mathrm{Mg}_{3.36} \mathrm{Fe}^{3+}{ }_{0.87} \mathrm{Fe}^{2+}{ }_{0.13} \mathrm{Ti}_{0.60}$ & $\mathrm{Si}_{5.92} \mathrm{Al}_{2.08}$ & $\mathrm{O}_{22}$ & $\mathrm{~F}_{1.11} \mathrm{O}_{0.89}$ \\
\hline K-Mg-Hs, Ylmenogorsky complex, South Urals, Russia [18] & $\mathrm{K}_{0.65} \mathrm{Na}_{0.31} \mathrm{Ba}_{0.04}$ & $\mathrm{Ca}_{1.93} \mathrm{Mn}_{0.06} \mathrm{Ba}_{0.01}$ & $\mathrm{Mg}_{2.32} \mathrm{Fe}^{2+}{ }_{1.12} \mathrm{Fe}^{3+}{ }_{0.84} \mathrm{Al}_{0.47} \mathrm{Ti}_{0.17}$ & $\mathrm{Si}_{5.53} \mathrm{Al}_{2.47}$ & $\mathrm{O}_{22}$ & $(\mathrm{OH})_{1.93} \mathrm{Cl}_{0.13}$ \\
\hline K-Mg-Hs, Prince Olav Coast, East Antarctica [17] & $\mathrm{K}_{0.85} \mathrm{Na}_{0.11} \mathrm{Ca}_{0.02}$ & $\mathrm{Ca}_{1.98} \mathrm{Mn}_{0.02}$ & $\mathrm{Mg}_{1.92} \mathrm{Fe}^{2+}{ }_{1.20} \mathrm{Fe}^{3+}{ }_{1.11} \mathrm{Al}_{0.64} \mathrm{Ti}_{0.13} \mathrm{Mn}_{0.01}$ & $\mathrm{Si}_{5.64} \mathrm{Al}_{2.36}$ & $\mathrm{O}_{22}$ & $(\mathrm{OH})_{1.36} \mathrm{O}_{0.48} \mathrm{~F}_{0.12} \mathrm{Cl}_{0.04}$ \\
\hline O-Mg-Hs, Deeti volcanic cone, Northern Tanzania [21] & $\mathrm{Na}_{0.67} \mathrm{~K}_{0.33}$ & $\mathrm{Ca}_{1.87} \mathrm{Na}_{0.14} \mathrm{Mn}_{0.01}$ & $\mathrm{Mg}_{3.27} \mathrm{Fe}^{3+}{ }_{1.25} \mathrm{Ti}_{0.44} \mathrm{Al}_{0.08}$ & $\mathrm{Si}_{6.20} \mathrm{Al}_{1.80}$ & $\mathrm{O}_{22}$ & $\mathrm{O}_{1.40}(\mathrm{OH})_{0.60}$ \\
\hline
\end{tabular}


The projection of the crystal structure parallel to the $a$-axis is shown in Figure 4 . The potassic-hastingsite structure is based on the infinite chain of $\mathrm{T}_{4} \mathrm{O}_{11}$ anions. The sixcoordinated $C$ cations sandwiched between two chains occupy the positions marked $M 1$, $M 2$, and $M 3$, arranging as a strip. The B cations occupy the $M 4$ positions at the edge of the whole tetrahedral-octahedral complex. The A cations lie between the backs of the chains.

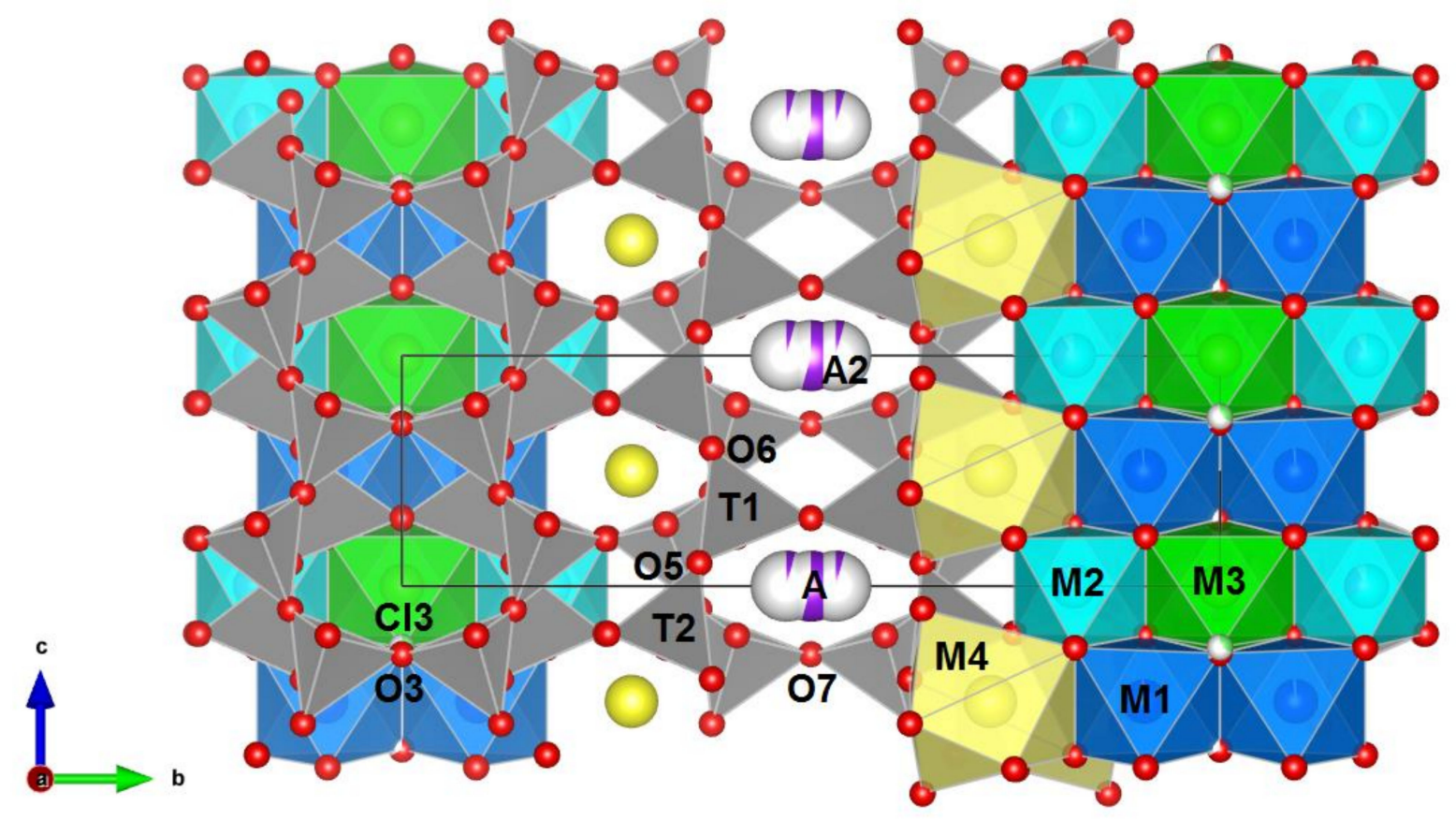

Figure 4. Crystal structure of potassic-hastingsite projected along the $a$ axis. Oxygen atoms are drawn in red. Si-tetrahedra and mixed Si-Al tetrahedra are gray. M1-blue, M2-cyan, M3-green octahedra, M4-yellow polyhedra. A and A2 positions are designated as lilac spheres. $\mathrm{Cl} 3$ sites are drawn in light green. The partially white coloring of the spheres indicates a vacancy. The relations between the ribbon of octahedra and the double-chain tetrahedra are shown.

The unit-cell dimensions for studied single crystal of potassic-hastingsite are $a=9.9724(3) \AA, b=18.2968(4) \AA, c=5.3573(1) \AA, \beta=104.945(3)^{\circ}, V=944.44(4) \AA^{3}, Z=2$. Final atomic coordinates, site occupancies, and equivalent and anisotropic displacement parameters are reported in Table 6 and Table S1 of the Supplementary Materials. In Table 7, selected bond lengths, angles, and distortion parameters describing the coordination polyhedra for the studied sample are provided.

Table 6. Crystallographic coordinates, occupancies, and equivalent/isotropic atomic displacement parameters $\left(\AA^{2}\right)$ of the studied potassic-hastingsite sample.

\begin{tabular}{ccccccc}
\hline Site & Atom & $x / a$ & $y / b$ & $z / c$ & Occ. & Ueq \\
\hline$A$ & $\mathrm{~K}^{+}$ & 0 & 0.5 & 0 & $0.524(1)$ & 0.0499 \\
$A 2$ & $\mathrm{~K}^{+}$ & 0 & $0.4728(7)$ & 0 & $0.037(1)$ & 0.0499 \\
$M 1$ & $\mathrm{Fe}^{2+}$ & 0 & $0.09259(2)$ & 0.5 & 1 & 0.0144 \\
$M 2$ & $\mathrm{Fe}^{2+}, \mathrm{Fe}^{3+}, \mathrm{Mg}^{2+}$ & 0 & $0.17915(2)$ & 0 & $0.99(1)$ & 0.119 \\
$M 3$ & $\mathrm{Fe}^{2+}$ & 0 & 0 & 0 & 1 & 0.0136 \\
$M 4$ & $\mathrm{Ca}^{2+}$ & 0 & $0.28067(2)$ & 0.5 & 1 & 0.0158 \\
$T 1$ & $\mathrm{Si}^{4+}, \mathrm{Al}^{3+}$ & $0.27837(4)$ & $0.08558(2)$ & $0.29999(8)$ & 1 & 0.0110 \\
$T 2$ & $\mathrm{Si}^{4+}$ & $0.29083(4)$ & $0.17265(2)$ & $0.80998(7)$ & 1 & 0.0108 \\
$\mathrm{O} 1$ & $\mathrm{O}^{2-}$ & $0.1057(1)$ & $0.09079(6)$ & $0.2138(2)$ & 1 & 0.0143 \\
$\mathrm{O} 2$ & $\mathrm{O}^{2-}$ & $0.1218(1)$ & $0.17736(6)$ & $0.7320(2)$ & 1 & 0.0138 \\
$\mathrm{O} 3$ & $\mathrm{O}^{2-}, \mathrm{F}^{-}$ & $0.1092(5)$ & 0 & $0.7087(4)$ & $0.68(1)$ & 0.0135 \\
& $\mathrm{Cl}^{-}$ & $0.1623(2)$ & 0 & $0.7358(3)$ & $0.31(1)$ & 0.0154 \\
\hline
\end{tabular}


Table 6. Cont.

\begin{tabular}{ccccccc}
\hline Site & Atom & $x / \boldsymbol{a}$ & $y / \boldsymbol{b}$ & $z / \boldsymbol{c}$ & Occ. & Ueq \\
\hline O4 & $\mathrm{O}^{2-}$ & $0.3688(1)$ & $0.24841(6)$ & $0.7935(2)$ & 1 & 0.0159 \\
$\mathrm{O} 5$ & $\mathrm{O}^{2-}$ & $0.3467(1)$ & $0.13651(6)$ & $0.1003(2)$ & 1 & 0.0155 \\
O6 & $\mathrm{O}^{2-}$ & $0.3423(1)$ & $0.12116(6)$ & $0.5967(2)$ & 1 & 0.0162 \\
O7 & $\mathrm{O}^{2-}$ & $0.3331(2)$ & 0 & $0.2953(3)$ & 1 & 0.0195 \\
\hline
\end{tabular}

Table 7. Selected bond distances $(\AA)$, angles and calculated geometrical and distortion parameters for tatrahedra, octahedra and polyhedra in the crystal structure of the studied potassic-hastingsite sample. The volume of the coordination tatrahedra, octahedra and polyhedra (Vt, Vo and Vp), as well as bond length distortion (BLD), tetrahedral and octahedral angle variance (TAV and OAV) and tetrahedral and octahedral quadratic elongation (TQE and OQE) were calculated using VESTA (version 4.3.0) [39] according [43,44].

\begin{tabular}{|c|c|c|c|c|c|}
\hline \multicolumn{2}{|c|}{ T1 Site } & \multicolumn{2}{|c|}{ T2 Site } & \multicolumn{2}{|c|}{$A$ Site } \\
\hline T1-O1 & $1.667(1)$ & $\mathrm{T} 2-\mathrm{O} 2$ & $1.631(1)$ & $A-\mathrm{O} 5(\times 4)$ & $3.048(1)$ \\
\hline T1-O5 & $1.689(1)$ & $\mathrm{T} 2-\mathrm{O} 4$ & $1.603(1)$ & $A-\mathrm{O} 6(\times 4)$ & $3.210(1)$ \\
\hline T1-O6 & 1.684(1) & T2-O5 & 1.650(1) & $A-\mathrm{O} 7(\times 2)$ & $2.576(2)$ \\
\hline T1-O7 & $1.660(1)$ & T2-O6 & $1.661(1)$ & $<A-\mathrm{O}>$ & $3.018(3)$ \\
\hline \multirow[t]{2}{*}{$<T 1-\mathrm{O}>$} & $1.675(3)$ & $<\mathrm{T} 2-\mathrm{O}>$ & $1.636(4)$ & & \\
\hline & $110.49(5)$ & $\mathrm{O} 2-\mathrm{T} 2-\mathrm{O} 4$ & $115.13(6)$ & $\begin{array}{l}\operatorname{Vp}\left(\AA^{3}\right) \\
\operatorname{BLD}(\%)\end{array}$ & $\begin{array}{c}50.300 \\
5.866\end{array}$ \\
\hline O1-T1-O6 & $110.59(6)$ & $\mathrm{O} 2-\mathrm{T} 2-\mathrm{O} 5$ & 109.73(6) & \multicolumn{2}{|c|}{$A 2$ site } \\
\hline O1-T1-O7 & $111.71(7)$ & $\mathrm{O} 2-\mathrm{T} 2-\mathrm{O} 6$ & 109.64(6) & $\mathrm{A} 2-\mathrm{O} 5(\times 2)$ & $2.614(25)$ \\
\hline O5-T1-O6 & $105.54(8)$ & O4-T2-O5 & $110.36(6)$ & A2-O6 (×2) & $2.856(19)$ \\
\hline O5-T1-O7 & $108.85(7)$ & $\mathrm{O} 4-\mathrm{T} 2-\mathrm{O} 6$ & $102.27(6)$ & A2-O7 (×2) & $2.635(7)$ \\
\hline O6-T1-O7 & 109.44(7) & O5-T2-O6 & $109.39(6)$ & $<\mathrm{A} 2-\mathrm{O}>$ & $2.702(48)$ \\
\hline$<\mathrm{O}-\mathrm{T} 1-\mathrm{O}>$ & $109.4(2)$ & $<\mathrm{O}-\mathrm{T} 2-\mathrm{O}>$ & 109.4(1) & \multicolumn{2}{|c|}{ M3 site } \\
\hline T1-O7-T1 & $141.15(12)$ & T1-O5-T2 & $136.49(7)$ & $\begin{array}{l}\text { M3-O1 }(\times 4) \\
\text { M3-O3 }(\times 2) \\
\text { M3-Cl3 }(\times 2)\end{array}$ & $\begin{array}{l}2.135(1) \\
2.121(4) \\
2.409(3)\end{array}$ \\
\hline $\operatorname{Vt}\left(\AA^{3}\right)$ & 2.407 & $\mathrm{Vt}\left(\AA^{3}\right)$ & 2.233 & $<M 3-\mathrm{O}_{6}>$ & $2.130(5)$ \\
\hline BLD (\%) & 0.684 & BLD (\%) & 1.176 & $<\mathrm{M} 3-\mathrm{O}_{5} \mathrm{Cl}>$ & $2.178(3)$ \\
\hline TAV & 4.6077 & TAV & 16.9950 & $<\mathrm{M} 3-\mathrm{O}_{4} \mathrm{Cl}_{2}>$ & $2.226(5)$ \\
\hline TQE & 1.0011 & TQE & 1.0044 & \multicolumn{2}{|c|}{ M4 site } \\
\hline \multicolumn{2}{|c|}{ M1 site } & \multicolumn{2}{|c|}{ M2 site } & M4-O2 $(\times 2)$ & $2.412(1)$ \\
\hline M1-O1 (×2) & $2.073(1)$ & M2-O1 $(\times 2)$ & $2.101(1)$ & M4-O4 $(\times 2)$ & $2.351(1)$ \\
\hline M1-O2 (×2) & $2.156(1)$ & M2-O2 $(\times 2)$ & $2.107(1)$ & M4-O5 $(\times 2)$ & $2.744(1)$ \\
\hline M1-O3 $(\times 2)$ & 2.163(1) & M2-O4 $(\times 2)$ & $1.986(1)$ & M4-O6 $(\times 2)$ & $2.527(1)$ \\
\hline $\mathrm{M} 1-\mathrm{Cl} 3(\times 2)$ & $2.456(1)$ & $<M 2-\mathrm{O}>$ & $2.065(3)$ & $<M 4-\mathrm{O}>$ & $2.508(3)$ \\
\hline$<M 1-\mathrm{O}_{6}>$ & $2.131(3)$ & & & & \\
\hline$<\mathrm{M} 1-\mathrm{O}_{5} \mathrm{Cl}>$ & $2.180(3)$ & $\operatorname{Vo}\left(\AA^{3}\right)$ & 11.611 & $\operatorname{Vp}\left(\AA^{3}\right)$ & 26.509 \\
\hline$<M 1-\mathrm{O}_{4} \mathrm{Cl}_{2}>$ & $2.228(3)$ & $\begin{array}{c}\text { BLD (\%) } \\
\text { OAV }\end{array}$ & $\begin{array}{c}2.532 \\
23.3661\end{array}$ & $\operatorname{BLD}(\%)$ & 5.069 \\
\hline$M 1-M 2$ & $3.1119(2)$ & OQE & 1.0078 & $A-A 2$ & $0.498(13)$ \\
\hline$M 1-M 3$ & $3.1694(2)$ & & & $A 2-A 2$ & $1.00(3)$ \\
\hline$M 2-M 3$ & $3.2779(4)$ & & & $\mathrm{O} 3-\mathrm{Cl} 3$ & $0.511(3)$ \\
\hline
\end{tabular}

\subsection{In Situ HTXRPD}

Initial cell dimensions obtained from the powder data refinement are as follows: $a=9.975(2) \AA, b=18.282(5) \AA, c=5.364(2) \AA, \beta=104.97(3)^{\circ}$, and $V=944.9(4) \AA^{3}$ (at $30^{\circ} \mathrm{C}$ ), and are closed to those derived from SCXRD data. The unit cell parameters calculated at each step of heating and cooling are given in Table S2 of the Supplementary Materials. The color of the potassic-hastingsite powder changed from dark green to brown after the experiment. 
On heating and cooling from 30 to $750{ }^{\circ} \mathrm{C}$ and back to $30^{\circ} \mathrm{C}$, the peaks positions in the diffraction patterns changed smoothly (Figures S1 and S2, Table S3 of the Supplementary Meterials). The evolution of the unit cell parameters and volume of potassic-hastingsite as a function of temperature is plotted in Figures 5 and 6.

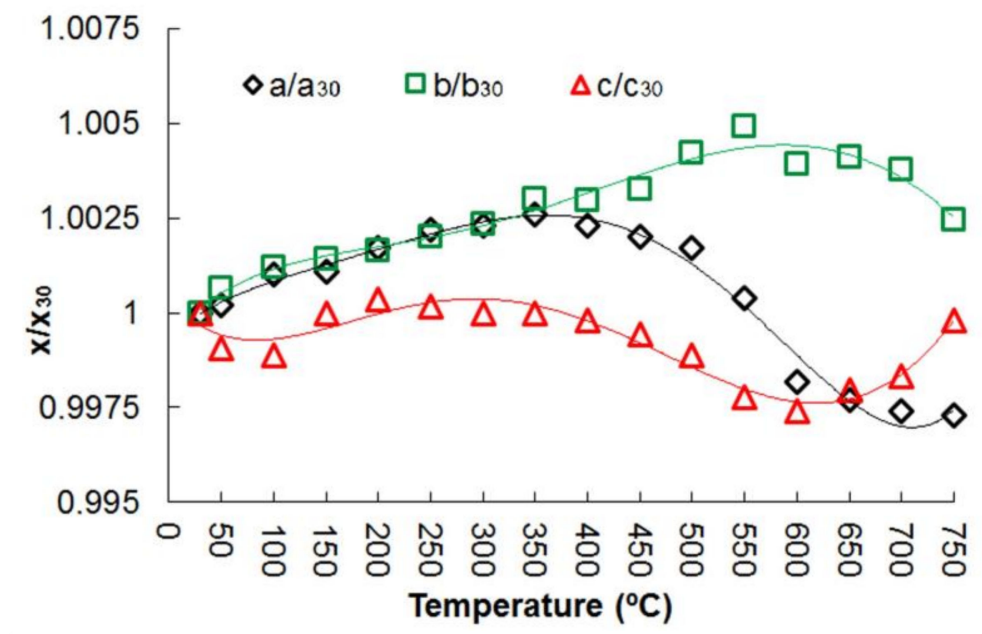

Figure 5. Normalized unit cell parameters of potassic-hastingsite $v$ s temperature. Symbols: $a / a_{30}$ black diamonds; $b / b_{30}$ - green squares; $c / c_{30}$-red triangles. Estimated standard deviations are smaller than the symbol used. A fifth-order polynomial approximation has been used for trend lines.

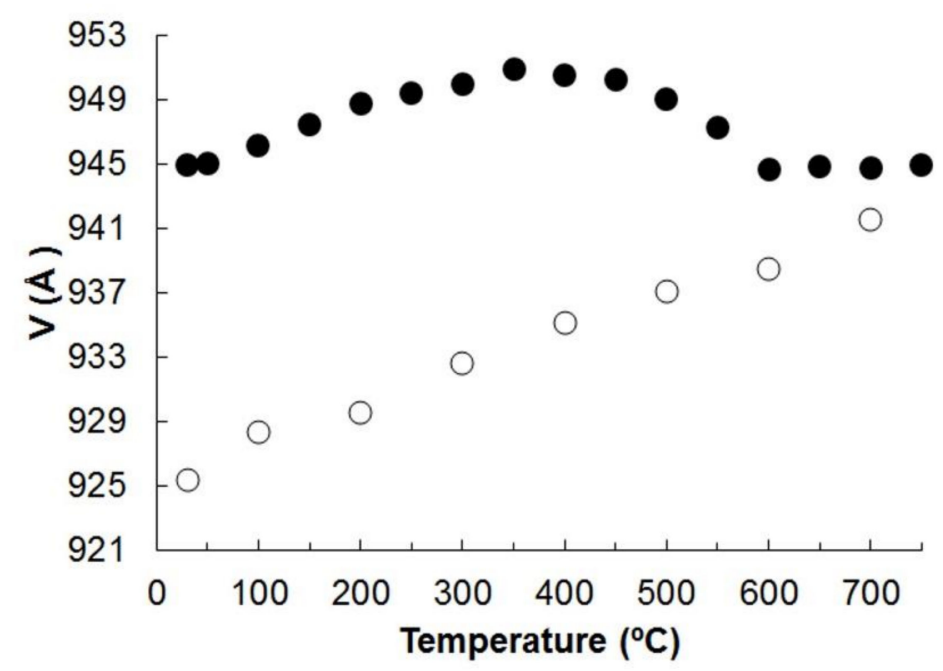

Figure 6. Unit cell volume of potassic-hastingsite vs temperature. Symbols: solid for the heating experiment; empty for the cooling. Estimated standard deviations are smaller than the symbol used.

The coefficients of thermal expansion tensor and the orientation of the principal axes of the thermal-expansion tensor with respect to the crystallographic axes are listed in Table S4 of the Supplementary Materials. A fifth-order polynomial approximation of temperature dependencies has been used.

\subsection{Spectroscopy}

Infrared absorption spectra of initial and annealed at $750{ }^{\circ} \mathrm{C}$ potassic-hastingsite are shown in Figure 7. Weak bands peaked at 595, 660, 739, $3610 \mathrm{~cm}^{-1}$ and strong bands at 877 , 935,968 , and $1049 \mathrm{~cm}^{-1}$ were observed in the initial potassic-hastingsite. During annealing most changes occur in the range $550-750 \mathrm{~cm}^{-1}$. Two bands near 595 and $730 \mathrm{~cm}^{-1}$ start to displace after heating to $550{ }^{\circ} \mathrm{C}$ together with the $\mathrm{OH}$ stretching band at $3610 \mathrm{~cm}^{-1}$ and disappear after sample heating at temperatures higher than $650{ }^{\circ} \mathrm{C}$. The $\mathrm{OH}$-stretching 
band demonstrates the same temperature behavior. A weak intensity band at $684 \mathrm{~cm}^{-1}$ is not retired, but it is displaced at $660 \mathrm{~cm}^{-1}$ during sample heating. The absorption spectrum of the sample that was annealed at $750{ }^{\circ} \mathrm{C}$ is given in Figure 7 (curve 2).

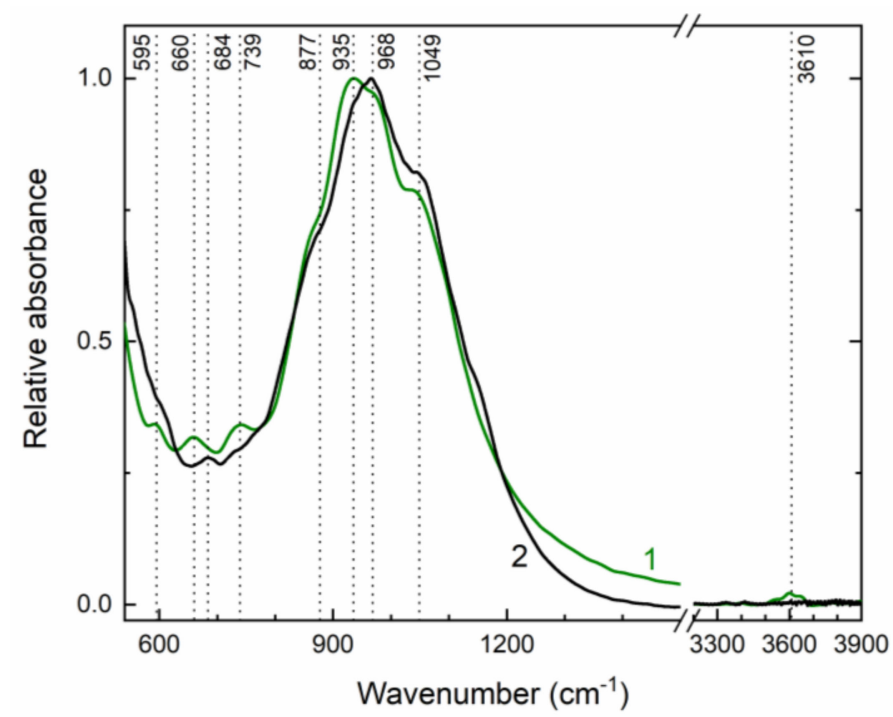

Figure 7. Infrared absorption spectra of initial (green curve 1) and annealed at $750{ }^{\circ} \mathrm{C}$ (black curve 2) potassic-hastingsite.

In the UV/Vis/NIR absorption spectrum of initial potassic-hastingsite, strong structureless bands at the 800-1600 $\mathrm{nm}$ spectral region are observed (Figure 8, curve 1). In addition, a moderate-intensity band in the range $520-800 \mathrm{~nm}$ is found. After annealing at $750{ }^{\circ} \mathrm{C}$ the absorption spectrum is changed. The strong band grows in the $250-600 \mathrm{~nm}$ spectral region, but the bands in the $800-1600 \mathrm{~nm}$ spectral region and at about $680 \mathrm{~nm}$ are decreased (Figure 8, curve 2).

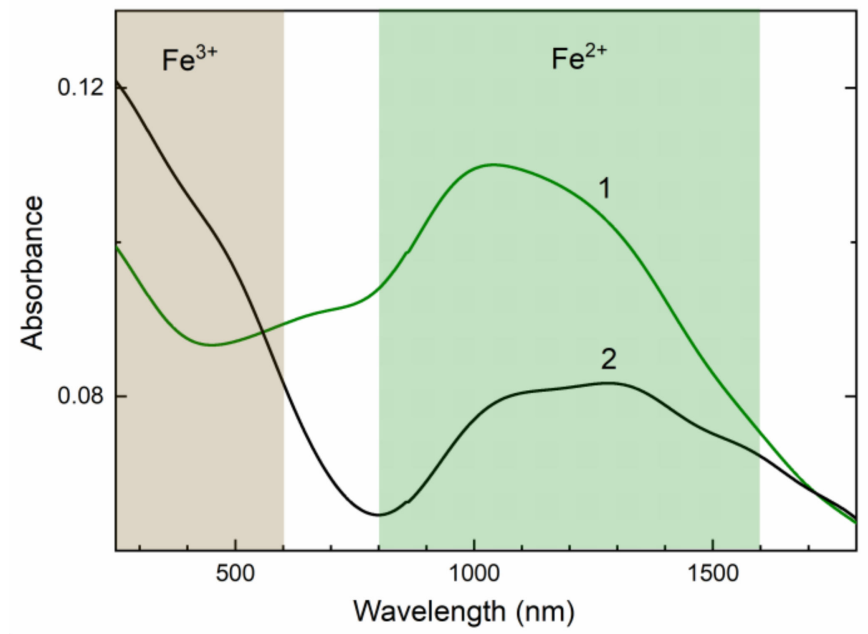

Figure 8. Diffuse light UV/Vis/NIR absorption spectra of initial (green curve 1) and annealed (black curve 2) potassic-hastingsite.

A strong ESR signal is detected at room temperature. ESR spectrum of the initial potassic-hastingsite is shown in Figure 9 (curve 1). Strong wide peaks with g-factors 4.2 and 2.01 are found in the spectrum. In the annealed at $750{ }^{\circ} \mathrm{C}$ sample, the intensity of the ESR signal is increased and changed in comparison with the initial sample (Figure 9, curve 2). Strong wide bands have g-factors at about 2.3 and 1.9. The relationship between the spectra of the annealed and the initial samples is shown in the inset to Figure 9. 


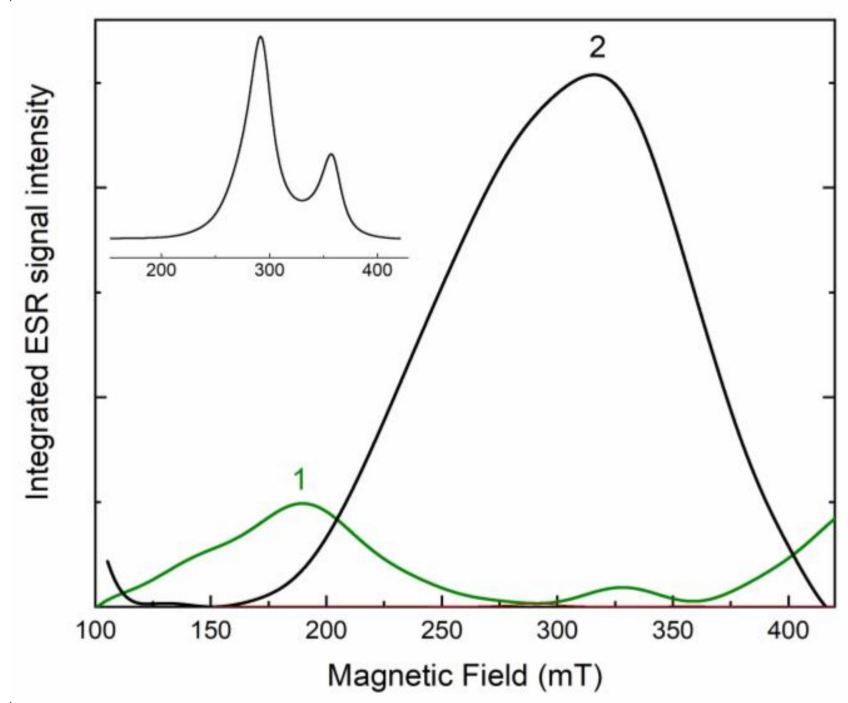

Figure 9. Electron spin resonance spectra of initial (green curve 1) and annealed (black curve 2) potassic-hastingsite. The ratio of curve 2 to curve 1 is given in the inset to the Figure.

\section{Discussion}

\subsection{Chrystal Chemical Features}

Table 8 yields the results of a bond-valence analysis for the studied sample. Analysis of Table 8 reveals that the bond-valence sums (BVS) are generally satisfactory for the oxygen atoms. A disordering of the anionic $\mathrm{O} 3$ site over two positions partially occupied by $\mathrm{OH}$-group and $\mathrm{Cl}$ ion is observed. The $\mathrm{O} 3$ site has a low bond-valence sum $(1.0 \mathrm{vu}$ (valence units)), leading to the conclusion that it is populated with $(\mathrm{OH})^{-}$and $\mathrm{F}^{-}$and does not contain $\mathrm{O}^{2-}$ atoms.

Table 8. Bond-valence sum for the studied potassic-hastingsite using the parameters by $[45,46]$.

\begin{tabular}{|c|c|c|c|c|c|c|c|c|c|}
\hline & $T 1$ & $T 2$ & $A$ & $A 2$ & M1 & M2 & M3 & M4 & $\sum$ \\
\hline O1 & 0.897 & & & & $0.395^{[\times 2]}$ & $0.371^{[\times 2]}$ & $0.344^{[\times 4]}$ & & 2.007 \\
\hline $\mathrm{O} 2$ & & 0.984 & & & $0.328^{[\times 2]}$ & $0.366^{[\times 2]}$ & & $0.291^{[\times 2]}$ & 1.969 \\
\hline $\mathrm{O} 3$ & & & & & $0.323^{[\times 2](\times 2)}$ & & $0.355^{[\times 2]}$ & & 1.001 \\
\hline $\mathrm{Cl} 3$ & & & & & $0.343^{[\times 2](\times 2)}$ & & $0.389^{[\times 2]}$ & & 1.075 \\
\hline $\mathrm{O} 4$ & & 1.055 & & & & $0.480^{[\times 2]}$ & & $0.337^{[\times 2]}$ & 1.872 \\
\hline O5 & 0.847 & 0.936 & $0.081^{[\times 4]}$ & $0.241^{[\times 2]}$ & & & & $0.129^{[\times 2]}$ & $1.993-2.153$ \\
\hline O6 & 0.858 & 0.909 & $0.054^{[\times 4]}$ & $0.131^{[\times 2]}$ & & & & $0.219^{[\times 2]}$ & $2.040-2.117$ \\
\hline O7 & $0.912^{(\times 2)}$ & & $0.265^{[\times 2]}$ & $0.228^{[\times 2]}$ & & & & & $2.089-2.052$ \\
\hline$\sum$ & 3.515 & 3.884 & 1.070 & 1.200 & $2.092-2.132$ & 2.434 & $2.086-2.154$ & 1.952 & \\
\hline
\end{tabular}

A cations. The A cations occur at the center of a large cavity between the two tetrahedral chains, oriented back-to-back with respect to each other. In the crystal structure of the studied sample, A cation site is partially populated by $\mathrm{K}$ and $\mathrm{Na}$. It should be noted that the A cation position in the studied structure is split.

B cations. Ca and minor $\mathrm{Na}$ and $\mathrm{Mn}$ (1\% of the total occupancy for each, Table 5) are located at the $\mathrm{B}$ cations formula site and the $M 4$ structural position, within the eightcoordinated polyhedron. The bond valence sum for the atoms at $M 4$ positions is almost 2 (Table 8).

$C$ cations. The octahedral $M 1, M 2$, and $M 3$ cation sites are mainly occupied by $\mathrm{Fe}^{2+}$ and $\mathrm{Fe}^{3+}$ with minor amounts of $\mathrm{Mg}, \mathrm{Al}, \mathrm{Mn}$, and Ti. The BVS of of $\mathrm{M} 1$ and $\mathrm{M} 3$ sites equals $\sim 2.1 \mathrm{vu}$ (Table 8), whereas the sum of the bond valence at the $M 2$ site is $2.43 \mathrm{vu}$ (i.e., $2<$ vu $<3$; Table 8) and the average M2-O distance is 2.065(3) $\AA$ (Table 7) which gives grounds to assume that Fe enters the M2 of the crystal structure in both two- and three- 
valent form, as expected based on previous literature. This polyhedron is also noted by slightly lower octahedral volume and BLD parameter and slightly greater OAV (octahedral angle variance; [43]) distortion (see Table 7). $\mathrm{Fe}^{2+}$ occupies $M 1$ and $M 3$ positions, forming $\mathrm{Fe}^{2+}-\mathrm{Cl}^{-}$pairs, as reported by [47]. Oberti et al. (2007) have reported crystal-chemical relationships, which allow calculation of the oxo component using the refined M1-M2 distance in calcium amphiboles [37]. This method provides $0.6{ }^{\mathrm{W}} \mathrm{O}^{2-}$ apfu for studied structural model with $M 1-M 2=\sim 3.11 \AA$ (Table 7). However, O3 position splitting and significant ${ }^{M 2} \mathrm{Mg}$ content might affect the experimental interatomic distances and lead to higher estimates of the oxo component, which is inconsistent with the values calculated using the EPMA analysis.

T cations. The measured $T-\mathrm{O}$ individual distances range from 1.660(1) to 1.689(1) and from 1.603(1) to 1.661(1) for $T 1$ and $T 2$ sites, respectively. On the basis of observed $\langle T-O\rangle$ distances (Table 7), it is possible to state that the $T 1$ position $(<T 1-\mathrm{O}>\sim 1.68 \AA$; tetrahedral volume, $\mathrm{Vt}$, is equal to $2.41 \AA^{3}$; $\mathrm{BVS} \sim 3.5 \mathrm{vu}$ ) has mixed occupancy ( $\mathrm{Al} \sim 1.91$ apfu according to the equation published by [37]); whereas the $T 2$ site $\left(<\mathrm{T} 2-\mathrm{O}>\sim 1.64 \AA\right.$; Vt $\sim 2.23 \AA^{3}$; BVS $\sim 3.9 \mathrm{vu}$ ) is fully filled by $\mathrm{Si}^{4+}$ ion, which is a common for monoclinic amphiboles [48] The BLD values for $T 1$ and $T 2$ tetrahedra are $\sim 0.68 \%$ and $~ 1.18 \%$, respectively, and the TAV (tetrahedral angle variance; [44]) values are $\sim 4.61$ and $\sim 17.00$, respectively (Table 7).

\subsection{Thermal Behavior and Spectroscopic Properties}

No evidence of phase transition was observed within the $T$ range investigated and all the diffraction patterns were successfully indexed in the $C 2 / m$ space group. The unit cell volume variation as a function of $\mathrm{T}$ is shown in Figure 6 . The measured evolution of the unit cell parameters is shown in Figure 5.

The thermal expansion of potassic-hastingsite is anisotropic. The graphical representation of the thermal expansion of the potassic-hastingsite is displayed in Figures S3-S7 of the Supplementary Materials. At $\mathrm{T}=100-300{ }^{\circ} \mathrm{C}$ the mineral exhibits a positive expansion for all the directions (Figure 6 and Figure S3 of the Supplementary Materials) (the thermal expansivity coefficients are $\alpha_{a}: \alpha_{b}: \alpha_{c}\left(\times 10^{-6}\right)=2.34: 9.26: 1.15$ at $100{ }^{\circ} \mathrm{C}$ and $-0.75: 7.11: 5.58$ at $300{ }^{\circ} \mathrm{C}$ ), while a shrinkage along the [101] direction occurs at $\mathrm{T}=350{ }^{\circ} \mathrm{C}$ (Figure $\mathrm{S} 4$ of the Supplementary Materials). The thermal expansivity coefficients are $\alpha_{a}: \alpha_{b}: \alpha_{c}\left(\times 10^{-6}\right)=-9.41: 8.71: 2.32$ at $350{ }^{\circ} \mathrm{C}$. In the temperature range 400-600 ${ }^{\circ} \mathrm{C}$ potassic-hastingsite shows a strong negative thermal expansion (Figure 6 and Figures S5 and S6 of the Supplementary Materials). The thermal expansivity coefficients are $\alpha_{a}: \alpha_{b}: \alpha_{\mathcal{C}}\left(\times 10^{-6}\right)=-18.06: 9.59:-1.09$ at $400{ }^{\circ} \mathrm{C}$ and $-26.15:-1.52: 2.22$ at $600{ }^{\circ} \mathrm{C}$. Starting from $600{ }^{\circ} \mathrm{C}$, the mineral expands again preferentially along [001] and shrinks along [110] without changes in the volume of unit cell (Figure 6 and Figure S7 of the Supplementary Materials). The thermal expansivity coefficients are $\alpha_{a}: \alpha_{b}: \alpha_{c}\left(\times 10^{-6}\right)=-20.86:-8.33:-10.56$ at $650{ }^{\circ} \mathrm{C}$ and 23.77:-25.06:42.08 at $750{ }^{\circ} \mathrm{C}$.

A closer comparison between the elastic properties of potassic-hastingsite can be made with amphiboles, Fe-rich holmquistite, riebeckite, investigated by [49] and [50,51], respectively, and synthetic potassic-ferro-richterite, studied by [52,53].

Oberti et al. (2018) reported that oxidation of $\mathrm{Fe}^{2+}$ and the consequent deprotonation at the $\mathrm{O} 3$ site causing the reduction of all unit cell parameters and volume in riebeckite starts at $\sim 350-425^{\circ} \mathrm{C}$ [50]. The temperature of the onset of deprotonation of potassicferro-richterite is even lower $\left(\sim 226^{\circ} \mathrm{C}\right.$; [52]). According to [50] and [52], iron oxidation in riebeckite and potassic-ferro-richterite takes place almost exclusively at the $M(1)$ site despite the fact that both the $M 1$ and $M 3$ polyhedra coordinate $(\mathrm{OH})$-anion at the $\mathrm{O} 3$ site.

In the range $30-350{ }^{\circ} \mathrm{C}$, our sample of potassic-hastingsite shows positive and linear expansion, with a small contraction of the $c$ parameter and $\beta$ angle. Taking into account the results of previous studies of monoclinic $\mathrm{Fe}^{2+}-$ and $\mathrm{Fe}^{3+}$-containing amphiboles [50,52], we concluded that the oxidation of Fe occurs in the temperature range of $400-600{ }^{\circ} \mathrm{C}$ when the unit cell volume decreases; then the $c$ parameter expands again (Figures 5 and 6). This value is somewhat higher than that observed for the aforementioned amphiboles. 
During the heating of studied potassic-hastingsite $\mathrm{Fe}^{2+}$ in the M1 position oxidizes to $\mathrm{Fe}^{3+}$, meanwhile $\mathrm{H}_{2}$ is released from the $\mathrm{O} 3$ site, common for two M1 and one M3 octahedra.

The $a$ edge is very sensitive to thermal expansion, followed by the $b$ edge (Figure 5). Moreover, the $a$ edge is the most sensitive to deprotonation (Figure 5). It can be noted that the thermal expansion process is completed at about $350{ }^{\circ} \mathrm{C}$, and after that the compression process of $a$ and $c$ unit cell parameters begins. After passing a temperature of $600{ }^{\circ} \mathrm{C}$, the $c$ increases again.

The sharp band at $3610 \mathrm{~cm}^{-1}$ on the IR spectrum (Figure 7) is assigned to the local configuration of ${ }^{\mathrm{M} 1} \mathrm{Fe}^{2+}{ }^{\mathrm{M} 1} \mathrm{Fe}^{2+}{ }^{2}{ }^{2} \mathrm{Fe}^{2+}-\mathrm{OH}-\mathrm{A}$ vacancy [54,55]. This band indicated that the studied amphibole has an ordered structure, having $\mathrm{Fe}^{2+}$ in the $\mathrm{M} 1$ and $\mathrm{M} 3$ positions and $\mathrm{Fe}^{3+}$ in the M2 position [56,57]. This spectroscopic data confirm the conclusions obtained from the SCXRD study and represented in Table 6.

The 595 and $730 \mathrm{~cm}^{-1}$ bands disappear in the IR spectrum of the annealed potassichastingsite. Their destruction is accompanied by a decrease in the intensity of the peak in the $3610 \mathrm{~cm}^{-1}$ region associated with the $\mathrm{OH}$ stretching vibrations. Thus, it can be assumed that the hydroxyl anion is also involved in the vibrations associated with the 595 and $730 \mathrm{~cm}^{-1}$ bands, and the deprotonation was complete. Early Ishida (1990) founded a broad or medium intensity $\mathrm{OH}$ librational band near $600 \mathrm{~cm}^{-1}$ in A site-occupied and $\mathrm{Al}(\mathrm{IV})$-free amphiboles [58]. Sergeeva et al. (2020) pointed, that the infrared bands at 740 and $725 \mathrm{~cm}^{-1}$ correspond to librational vibrations of water coordinated to $\mathrm{Al}$ [59]. Generally, libration modes have been discussed in more detail by us in [60].

A medium intensity band at $684 \mathrm{~cm}^{-1}$ could be ascribed assigned to $\mathrm{O}_{\mathrm{nbr}}-\mathrm{Si}-\mathrm{O}_{\mathrm{nbr}}$ bending deformations following Ca- and other amphiboles [61]. The frequency of this band significantly depends on the octahedral cations and the distance between cation and O2 [58]. Therefore, after the high-temperature experiment the unit cell volume is decreased and the frequency of this bending vibration is shifted to the low energy region.

In the visible spectral region, the amphibole, cordierite, and sekaninaite absorption spectra display an intense pleochroic band located at about 580-750 nm attribute to $\mathrm{Fe}^{2+} / \mathrm{Fe}^{3+}$ intervalence charge transfer transition (IVCT) [62,63]. This charge transfer transition occurs between intervalence Fe pairs due to delocalization of the d-electron along $\mathrm{Fe}^{2+}-\mathrm{Fe}^{3+}$ bond.

Near-infrared absorption (NIR) bands are attributed to dd transitions in $\mathrm{Fe}^{2+}$ ions. The $\mathrm{Fe}^{2+}$ ion has a d ${ }^{6}$ electron configuration. In octahedral amphiboles absorption at about $1000 \mathrm{~nm}$ is due to transition in $\mathrm{Fe}^{2+}$ cations in position M1 (Figure 8). Longer wavelength absorption bands at 1300 and $1600 \mathrm{~nm}$ are also found in Ca-amphiboles. They are caused by spin-allowed dd transition of $\mathrm{Fe}^{2+}$ in $\mathrm{M} 2$ and M3 sites [62,64].

In the annealed sample, the band in the region $250-600 \mathrm{~nm}$ appears (Figure 8). This absorption is assigned to dd transition in octahedral coordinated $\mathrm{Fe}^{3+}$ ions [65]. This is also led to changing the color of the potassic-hastingsite from green to brown.

Obtained ESR spectra are attributed to $\mathrm{Fe}^{3+}$ ions. In the initial sample, the ESR signal (Figure 9) with g-factors 2.01 and 4.2 corresponds to $\mathrm{Fe}^{3+}$ in a low spin octahedral position. The lines are highly broadened due to the exchange interaction between Fe ions [66]. In the annealed sample, the ESR signal is increased due to the appearance of two new components with g-factors 1.9 and 2.3 (Figure 9). A similar signal is usually registered for $\mathrm{Fe}^{3+}$ in high spin states in octahedral crystal field [67]. This fact is evidence that $\mathrm{Fe}^{3+}$ cations appear in new coordination after annealing of the potassic-hastingsite.

The change of absorption spectra occurs due to a decrease of $\mathrm{Fe}^{2+}$ concentration preferably in the M1 site because intensities of 1300 and 1600 bands are retained (Figure 8). At the same time, the concentration of $\mathrm{Fe}^{3+}$ cations is increased in the annealed sample. Based on ESR data, the position of these $\mathrm{Fe}^{3+}$ cations is the non-equivalent of the one in the initial sample (Figure 9). The diminution of the IVCT band in the $680 \mathrm{~nm}$ region corresponds to a decrease of $\mathrm{Fe}^{2+} / \mathrm{Fe}^{3+}$ pairs. 
The removal of $\mathrm{OH}$ bands by heating in air suggests that $\mathrm{OH}^{-}$(Figure 7) is lost through an oxidation-dehydrogenation reaction of the type [68]:

$$
\mathrm{Fe}^{2+}+\mathrm{OH}^{-} \rightarrow \mathrm{Fe}^{3+}+\mathrm{O}^{2-}+1 / 2 \mathrm{H}
$$

Therefore, the oxidation process leads to an increase in $\mathrm{Fe}^{3+}$ in the $M 1$ position where the $\mathrm{Fe}^{2+}$ cations are located before the heating. This oxidation is confirmed by the optical spectra, which show the decrease in $\mathrm{Fe}^{2+}$ content and increase of $\mathrm{Fe}^{3+}$ content. Furthermore, $\mathrm{Fe}^{2+}$ cations in the $M 1$ site participate in $\mathrm{Fe}^{2+} / \mathrm{Fe}^{3+}$ pairs, which provided an IVCT absorption band. After oxidation of $\mathrm{Fe}^{2+}$ IVCT absorption is diminished.

Finally, the values of the cell parameters refined at room temperature after the cooling of the sample are not the same obtained before the direct experiment (see Table S2 of the Supplementary Materials), indicating that the processes occurring in potassic-hastingsite during HT experiments are irreversible. Consistently, the HTXRPD data detect a cell volume decrease of the cooling phase of about $2 \%$ with respect to the initial one.

\section{Conclusions}

Amphiboles are an important supergroup of rock-forming minerals, which crystallize under almost all conditions and have unusually high chemical variability. They play a significant role in a number of metasomatic and metamorphic processes. The chemical diversity originates from their crystal structure, which is able to include a wide range of elements. Because of their importance, a number of mineralogical and spectroscopic investigations and studies at high temperature (HT) are constantly being carried out to describe the crystal-chemical and optical properties, thermoelastic behavior, and deformation mechanisms of the amphiboles.

Crystal chemistry, spectroscopy and thermal behavior of potassic-hastingsite from the Kedrovy district (East Siberia, Russia) with crystal-chemical formula ${ }^{\mathrm{A}}\left(\mathrm{K}_{0.46} \mathrm{Na}_{0.22}\right)$ ${ }^{\mathrm{B}}\left(\mathrm{Ca}_{1.95} \mathrm{Na}_{0.03} \mathrm{Mn}_{0.02}\right)^{\mathrm{C}(\mathrm{M} 1)}\left(\mathrm{Fe}^{2+}{ }_{1.96} \mathrm{Mn}_{0.04}\right)^{\mathrm{C}(\mathrm{M} 2)}\left(\mathrm{Fe}^{3+}{ }_{1.15} \mathrm{Fe}^{2+}{ }_{0.52} \mathrm{Mg}_{0.24} \mathrm{Al}_{0.06} \mathrm{Ti}_{0.02}\right)^{\mathrm{C}(\mathrm{M} 3)}\left(\mathrm{Fe}^{2+}\right)$ ${ }^{\mathrm{T}}\left(\mathrm{Si}_{6.01} \mathrm{Al}_{1.99}\right) \mathrm{O}_{22}{ }^{\mathrm{W}}\left(\mathrm{OH}_{1.33} \mathrm{Cl}_{0.58} \mathrm{~F}_{0.09}\right)$ were studied and the petrographic description of the amphibole-containing rock sample was carried out.

This is the first study in which the thermal behavior of potassic-hastingsite is described at temperatures up to $750^{\circ} \mathrm{C}$. The study indicates that this mineral undergoes a process of the oxidation of $\mathrm{Fe}^{2+}$ and the associated deprotonation that are complete and irreversible. In particular, the thermal experiment indicates that there are two steps of thermal evolution: expansion in the range $\left.30-350{ }^{\circ} \mathrm{C}\right)$ followed by the structural contraction $\left(\sim 400-600{ }^{\circ} \mathrm{C}\right)$ associated with oxidation of $\mathrm{Fe}^{2+}$ to $\mathrm{Fe}^{3+}$, while $\mathrm{H}_{2}$ is released. The spectroscopic studies confirm this statement. After heating, obtained optical spectra show the decrease in $\mathrm{Fe}^{2+}$ content and increase of $\mathrm{Fe}^{3+}$ content in the sample. The ESR evidences the appearance of $\mathrm{Fe}^{3+}$ in the new structural site.

The study of the mineral composition of the rock sample by petrographic methods makes it possible to assign it to Ca-Fe-Si postmagmatic skarns [69-72]. Paragenetic relationships of grossular-andradite, apatite, potassic-hastingsite, calcite, and vesuvianite (first generation) suggest that they were formed at the late hydrosilicate stage of skarn formation, occurring at temperatures of $\sim 500-400{ }^{\circ} \mathrm{C}$ [72-75] (Table 3). The mineral associations of the early stage are superimposed by hydrothermal parageneses: quartz, vesuvianite (second generation), and sulfide veinlets, epidote, and zoisite (Table 3). Thus, at the final hydrothermal stage (temperature $\sim 400-150^{\circ} \mathrm{C}$ ), the skarn underwent changes. However, the irreversible deprotonation and oxidation of iron, and as a consequence, the color change that occurs when potassic-hastingsite is heated above $400{ }^{\circ} \mathrm{C}$, indicate that after the hydrosilicate and final hydrothermal stages of skarn formation, the rock did not undergo transformations under the influence of temperatures above the specified range. It had a retrograde character. The transformation of sulfides into iron hydroxides took place, most likely, at the supergene stage of rock alteration. 
Supplementary Materials: The following are available online at https: / www.mdpi.com/article/ $10.3390 / \min 11101049 / \mathrm{s} 1$, Table S1: Anisotropic atomic displacement parameters $\left(\AA^{2}\right)$ of potassichastingsite sample, Table S2: Unit cell parameters of the studied potassic-hastingsite as a function of T, Table S3: X-ray powder diffraction data vs temperature for studied potassic-hastingsite, Table S4: Coefficients of thermal expansion tensor $\left(\alpha_{\mathrm{ij}}\right)$ of the studied potassic-hastingsite as a function of $\mathrm{T}$ and orientation of its eigenvectors with respect to unit cell axes, Figure S1: Selected XRPD patterns of potassic-hastingsite showing the evolution of the diffraction patterns collected during the heating experiment, Figure S2: XRPD patterns of potassic-hastingsite showing the evolution of the diffraction patterns collected during the cooling experiment, Figure S3: Graphical representation of the thermal expansion tensor of the potassic-hastingsite at $100{ }^{\circ} \mathrm{C}$, Figure S4: Graphical representation of the thermal expansion tensor of the potassic-hastingsite at $350^{\circ} \mathrm{C}$, Figure S5: Graphical representation of the thermal expansion tensor of the potassic-hastingsite at $400^{\circ} \mathrm{C}$, Figure S6: Graphical representation of the thermal expansion tensor of the potassic-hastingsite at $600{ }^{\circ} \mathrm{C}$, Figure S7: Graphical representation of the thermal expansion tensor of the potassic-hastingsite at $750{ }^{\circ} \mathrm{C}$.

Author Contributions: Conceptualization, E.K.; methodology, E.K. and R.S.; investigation, E.K., T.R., R.S., V.C. and V.D.; writing-original draft preparation, E.K., T.R., R.S. and V.C.; writing-review and editing, E.K.; visualization, E.K., T.R. and R.S. All authors have read and agreed to the published version of the manuscript.

Funding: This research was supported by INRTU, grant number 15-RAS-2020 (Grant of the University Council). XRF and powder XRD were performed by the governmental assignment in terms of Project 0284-2021-0005.

Data Availability Statement: Not applicable.

Acknowledgments: The authors thank the staff of Sidorov Mineralogical Museum of INRTU, who kindly provided the sample studied in this work. The study was carried out using facilities of the Centers for Collective Use: "Center for isotopic-geochemical investigations" at the Vinogradov Institute of Geochemistry SB RAS, "Baikal analytical center for collective use" at the Favorsky Irkutsk Institute of Chemistry SB RAS, and the "Analytical Center for Multielement and Isotope Research" at the Sobolev Institute of Geology and Mineralogy SB RAS. We are grateful to reviewers for their valuable comments.

Conflicts of Interest: The authors declare no conflict of interest.

\section{References}

1. Hawthorne, F.C.; Oberti, R. Classification of the amphiboles. Rev. Mineral. Geochem. 2007, 67, 55-88. [CrossRef]

2. Leake, B.E.; Woolley, A.R.; Arps, C.E.S.; Birch, W.D.; Gilbert, M.C.; Grice, J.D.; Hawthorne, F.C.; Kato, A.; Kisch, H.J.; Krivovichev, V.G.; et al. Nomenclature of amphiboles: Report of the Subcommittee on amphiboles of the International Mineralogical Association Commission on New Minerals and Mineral Names. Mineral. Mag. 1997, 61, 295-321. [CrossRef]

3. Hawthorne, F.C.; Oberti, R.; Harlow, G.E.; Maresch, W.V.; Martin, R.F.; Schumacher, J.C.; Welch, M.D. Nomenclature of the amphibole supergroup. Am. Mineral. 2012, 97, 2031-2048. [CrossRef]

4. Adams, F.D.; Harrington, B.J. On a new alkali hornblende and a titaniferous andradite from the nepheline-syenite of Dungannon, Hastings County, Ontario. Am. J. Sci. 1896, 151, 210-218.

5. Makino, K.; Tomita, K. Cation distribution in the octahedral sites of hornblendes. Am. Mineral. 1989, 74, 1097-1105.

6. Makino, K.; Tomota, K.; Suwa, K. Effect of chlorine on the crystal structure of a chlorine-rich hastingsite. Mineral. Mag. 1993, 57, 677-685. [CrossRef]

7. Dick, L.A.; Robinson, G.W. Chlorine-bearing potassian hastingsite from a sphalerite skarn in southern Yukon. Can. Mineral. 1979, 17, 25-26.

8. Suwa, K.; Enami, M.; Horiuchi, T. Chlorine-rich potassium hastingsite from West Ongul Island, Lutzow-Holm Bay, East Antarctica. Mineral. Mag. 1987, 51, 709-714. [CrossRef]

9. Žáček, V. Potassium hastingsite and potassichhastingsite from garnet-Hedenbergite skarn at Vlastějovice, Czech Republic. Neues Jahrb. Mineral.-Abh. 2007, 184, 161-168. [CrossRef]

10. Ren, G.; Li, G.; Shi, J.; Gu, X.; Fan, G.; Yu, A.; Liu, Q.; Shen, G. Potassic-hastingsite, $\mathrm{KCa}_{2}\left(\mathrm{Fe}^{2+}{ }_{4} \mathrm{Fe}^{3+}\right)\left(\mathrm{Si}_{6} \mathrm{Al}_{6}\right) \mathrm{O}_{22}(\mathrm{OH})_{2}$, from the Keshiketeng Banner, Inner Mongolia, China: Description of the neotype and its implication. Mineral. Petrol. 2020, 114, 403-412. [CrossRef]

11. Lupulescu, M.V.; Rakovan, J.; Dyar, M.D.; Robinson, G.W.; Hughes, J.M. Fluoro-potassichastingsite from the Greenwood mine, Orange county, New York: A new end-member calcic amphibole. Can. Mineral. 2009, 47, 909-916. [CrossRef]

12. Krutov, G.A. Dashkesanite-A new chlorine-containing amphibole of the hastingsite group. Izvest. AN USSR Geol. Ser. 1936, 2-3, 341-373. (In Russian) 
13. Pekov, I.V.; Chukanov, N.V.; Nefedova, M.E.; Pushcharovsky, D.Y.; Rastsvetaeva, R.K. Chloro-potassichastingsite $\left(\mathrm{K}_{,} \mathrm{Na}\right) \mathrm{Ca}_{2}\left(\mathrm{Mg}, \mathrm{Fe}^{2+}\right)_{4}$ $\mathrm{Fe}^{3+}\left[\mathrm{Si}_{6} \mathrm{Al}_{2} \mathrm{O}_{22}\right](\mathrm{OH}, \mathrm{Cl})_{2}$ : Revalidation and the new name of dashkesanite. Zap. RMO 2005, 6, 31-36. (In Russian)

14. Billings, M. The chemistry, optics and genesis of the hastingsite group of amphiboles. Am. Mineral. 1928, 13, $287-296$.

15. Walitzi, E.M.; Walter, F. Verfeinerung der keistallstruktur eines basaltischen magnesio-hastingsites. Z. Kristallogr. 1981, 156, 197-208. (In German) [CrossRef]

16. Martin, R.F.; Alarie, É.; Minarik, W.G.; Wáczek, Z.; McCammon, C.A. Titanium-rich magnesio-hastingsite macrocrysts in a camptonite dike, Lafarge quarry, Montreal island, Québec: Early crystallization in a pseudo-unary system. Can. Mineral. 2016, 54, 65-78. [CrossRef]

17. Shiraishi, K.; Oba, T.; Suzuki, M.; Ishikawa, K. Subsilic magnesian potassium-hastingsite from the Prince Olav Coast, East Antarctica. Mineral. Mag. 1994, 58, 621-627. [CrossRef]

18. Korinevsky, V.G.; Korinevsky, E.V. Potassic-magnesiohastingsite $(\mathrm{K}, \mathrm{Na}) \mathrm{Ca}_{2}\left(\mathrm{Mg}_{,} \mathrm{Fe}^{2+}\right)_{4}\left(\mathrm{Fe}^{3+}, \mathrm{Al}_{,} \mathrm{Ti}\right)\left[\mathrm{Si}_{6} \mathrm{Al}_{2} \mathrm{O}_{22}\right](\mathrm{OH}, \mathrm{Cl})_{2}-\mathrm{The}$ new mineral species of amphiboles. Zap. RMO 2006, 2, 49-57. (In Russian)

19. Bojar, H.-P.; Walter, F. Fluoro-magnesiohastingsite from Dealul Uroi (Hunedoara county, Romania): Mineral data and crystal structure of a new amphibole end-member. Eur. J. Mineral. 2006, 18, 503-508. [CrossRef]

20. Aksenov, S.; Chukanov, N.V. The crystal structure of a fluorine-dominant titanium calcium amphibole from the Eifel paleovolcanic area, Germany. Z. Kristallogr. 2016, 231, 385-390. [CrossRef]

21. Zaitsev, A.N.; Avdontseva, E.Y.; Britvin, S.N.; Demény, A.; Homonnay, Z.; Jeffries, T.E.; Keller, J.; Krivovichev, V.G.; Markl, G.; Platonova, N.V.; et al. Oxo-magnesio-hastingsite, $\mathrm{NaCa}_{2}\left(\mathrm{Mg}_{2} \mathrm{Fe}^{3+}{ }_{3}\right)\left(\mathrm{Al}_{2} \mathrm{Si}_{6}\right) \mathrm{O}_{22} \mathrm{O}_{2}$, a new anhydrous amphibole from the Deeti volcanic cone, Gewgory rift, northern Tanzania. Mineral. Mag. 2013, 77, 2773-2792. [CrossRef]

22. Oberti, R.; Zema, M.; Boiocchi, M.; Tarantino, S.; Welch, M.D. HT-induced processes in monoclinic and orthorhombic amphiboles and their effects on thermodynamic models. Geophys. Res. Abstr. EGU2011 2011, 13, 11183.

23. Tribaudino, M.; Hovis, G.L.; Almer, C.; Leaman, A. Thermal expansion of minerals in the amphibole supergroup. Am. Mineral. 2022, in press. [CrossRef]

24. Drugov, G.M.; Sizykh, A.I.; Bulanov, V.A. Geological models of mica-bearing junction of the Slyudanskaya and Sogdioddonskaya groups of deposits in the Mamsk province. Bull. Irkutsk State Univ. Ser. Earth Sci. 2015, 13, 58-77.

25. Whitney, D.L.; Evans, B.W. Abbreviations for names of rock-forming minerals. Am. Mineral. 2010, 95, 185-187. [CrossRef]

26. Verkaeren, J. Les grenats biréfringents des skarns à magnétite de San Leone (Sardaigne SW). Bull. Soc. Franc. Mineral. Cristallogr. 1971, 94, 492-499. (In French) [CrossRef]

27. Lessing, P.; Standish, R.P. Zoned garnet from Crested Butte, Colorado. Am. Mineral. 1973, 58, 840-842.

28. Takeuchi, Y.; Haga, N. Optical anomaly and structure of silicate garnets. Proc. Jpn. Acad. 1976, 52, 228-231. [CrossRef]

29. Yang, S.-Y.; Zhang, R.-X.; Jiang, S.-Y.; Xie, J. Electron probe microanalysis of variable oxidation state oxides: Protocol and pitfalls. Geostand. Geoanal. Res. 2018, 42, 131-137. [CrossRef]

30. Finkelshtein, A.L.; Chubarov, V.M. X-ray fluorescence determination of $\mathrm{FeO} / \mathrm{Fe}_{2} \mathrm{O}_{3}{ }^{\text {tot }}$ ratio in igneous rocks. X-ray Spectrom. 2010, 39, 17-21. [CrossRef]

31. Chubarov, V.M.; Finkelshtein, A.L. Determination of divalent iron content in igneous rocks of ultrabasic, basic and intermediate compositions by a wavelength-dispersive X-ray fluorescence spectrometric method. Spectrochim. Acta B 2015, 107, 110-114. [CrossRef]

32. Chubarov, V.M.; Amosova, A.A.; Finkelshtein, A.L. Determination of iron and sulfur valence state in coal ashes by wavelengthdispersive X-ray fluorescence spectrometric technique. Spectrochim. Acta B 2020, 163, 105745. [CrossRef]

33. Chubarov, V.M.; Finkelshtein, A.L.; Suvorova, L.F.; Kostrovitsky, S.I. Determination of iron valence state in picroilmenites by electron probe microanalysis and x-ray fluorescence analysis. Zap. RMO 2012, 141, 83-91. (In Russian)

34. Bruker APEX2. Version 2014.11-0; Bruker AXS Inc.: Madison, WI, USA, 2014.

35. CrysAlis, PRO. Version 1.171.35.21; Agilent Technologies Ltd.: Yarnton, UK, 2018.

36. Betteridge, P.W.; Carruthers, J.R.; Cooper, R.I.; Prout, K.; Watkin, D.J. Crystals version 12: Software for guided crystal structure analysis. J. App. Cryst. 2003, 36, 1487. [CrossRef]

37. Oberti, R.; Hawthorne, F.C.; Cannillo, E.; Cámara, F. long-range order in amphiboles. Rev. Mineral. Geochem. 2007, 67, 125-171. [CrossRef]

38. Hawthorne, F.; Oberti, R. Amphiboles: Crystal structure. Rev. Mineral. Geochem. 2007, 67, 1-54. [CrossRef]

39. Momma, K.; Izumi, F. VESTA 3 for three-dimensional visualization of crystal, volumetric and morphology data. J. Appl. Cryst. 2011, 44, 1272-1276. [CrossRef]

40. Bruker: Topas V4. General Profile and Structure Analysis Software For Powder Diffraction Data; Bruker AXS Inc.: Karlsruhe, Germany, 2008.

41. Langreiter, T.; Kahlenberg, V. TEV-A program for the determination of the thermal expansion tensor from diffraction data. Crystals 2015, 5, 143-153. [CrossRef]

42. Locock, A.J. An Exel spreadsheet to classify chemical analyses of amphiboles following the IMA 2012 recommendations. Comput. Geosci. 2014, 62, 1-11. [CrossRef]

43. Robinson, K.; Gibbs, G.V.; Ribbe, P.H. Quadratic elongation: A quantitative measure of distortion in coordination polyhedra. Science 1971, 172, 567-570. [CrossRef] 
44. Renner, B.; Lehmann, G. Correlation of angular and bond length distortions in $\mathrm{TO}_{4}$ units in crystals. Z. Kristallogr. 1986, 175, 43-59. [CrossRef]

45. Gagnè, O.C.; Hawthorne, F.C. Comprehensive derivation of bond-valence parameters for ion pairs involving oxygen. Acta Cryst. 2015, B71, 562-578. [CrossRef] [PubMed]

46. Breese, N.E.; O'Keeffe, M. Bond-valence parameters for solid. Acta Cryst. 1991, B47, 192-197. [CrossRef]

47. Oberti, R.; Ungaretti, L.; Cannillo, E.; Hawthorne, F. The mechanism of $\mathrm{Cl}$ incorporation in amphibole. Am. Mineral. 1993, 78, 746-752.

48. Oberti, R.; Ungaretti, L.; Cannillo, E.; Hawthorne, F.; Memmi, I. Temperature-dependent Al order-disorder in the tetrahedral double chain of $C 2 / m$ amphiboles. Eur. J. Mineral. 1995, 7, 1049-1063. [CrossRef]

49. Oberti, R.; Boiocchi, M.; Zema, M. Thermoelasticity, cation exchange, and deprotonation in Fe-rich holmquistite: Toward a crystal-chemical model for the high-temperature behavior of orthorhombic amphiboles. Am. Mineral. 2019, 104, 1829-1839. [CrossRef]

50. Oberti, R.; Boiocchi, M.; Zema, M.; Hawthorne, F.; Redhammer, G.; Susta, U.; Della Ventura, G. The high-temperature behavior of riebeckite: Expansivity, deprotonation, selective Fe oxidation and a novel cation disordering scheme for amphiboles. Eur. J. Mineral. 2018, 30, 437-449. [CrossRef]

51. Della Ventura, G.; Mihailova, B.; Susta, U.; Castelli Guidi, M.; Marcelli, A.; Schlüter, J.; Oberti, R. The dynamics of Fe oxidation in riebeckite: A model for amphiboles. Am. Mineral. 2018, 103, 1103-1111. [CrossRef]

52. Oberti, R.; Boiocchi, M.; Zema, M.; Della Ventura, G. Synthetic potassic-ferro-richterite: 1. Composition, crystal structure refinement, and HT behavior by in operando single-crystal X-ray diffraction. Can. Mineral. 2016, 54, 353-369. [CrossRef]

53. Della Ventura, G.; Susta, U.; Bellatreccia, F.; Marcelli, A.; Redhammer, G.; Oberti, R. Deprotonation of Fe-domonant amphiboles: Single-crystal HT-FTIR spectroscopic studies of synthetic potassic-ferro-richterite. Am. Mineral. 2017, 102, 117-125. [CrossRef]

54. Iezzi, G.; Della Ventura, G.; Hawthorne, F.C.; Pedrazzi, G.; Robert, J.L.; Novembre, D. The $\left(\mathrm{Mg}^{\prime} \mathrm{Fe}^{2+}\right)$ substitution in ferriclinoholmquistite, $\square \mathrm{Li}_{2}\left(\mathrm{Mg}, \mathrm{Fe}^{2+}\right)_{3} \mathrm{Fe}^{3+}{ }_{2} \mathrm{O}_{22}(\mathrm{OH})_{2}$. Eur. J. Mineral. 2005, 17, 733-740. [CrossRef]

55. Ishida, K. Assignment of infrared $\mathrm{OH}$-stretching bands in calcic amphiboles through deuteration and heat treatment. Am. Mineral. 2006, 91, 871-879. [CrossRef]

56. Burns, R.G.; Strens, R.G.J. Infrared study of the hydroxyl bonds in clinoamphiboles. Science 1966, 153, 890-892. [CrossRef]

57. Della Ventura, G. Recent developments in the synthesis and characterization of amphiboles. Synthesis and crystal chemistry of richterite. Trends Mineral. 1992, 1, 153-192.

58. Ishida, K. Identification of infrared $\mathrm{OH}$ librational bands of talc-willemseite solid solutions and $\mathrm{Al}$ (IV)-free amphiboles through deuteration. Mineral. J. 1990, 15, 93-104. [CrossRef]

59. Sergeeva, A.V.; Zhitova, E.S.; Nuzhdaev, A.A.; Zolotarev, A.A.; Bocharov, V.N.; Ismagilova, R.M. Infrared and Raman spectroscopy of ammoniovoltaite, $\left(\mathrm{NH}_{4}\right)_{2} \mathrm{Fe}^{2+}{ }_{5} \mathrm{Fe}^{3+}{ }_{3} \mathrm{Al}\left(\mathrm{SO}_{4}\right)_{12}\left(\mathrm{H}_{2} \mathrm{O}\right)_{18}$. Minerals 2020, 10, 781. [CrossRef]

60. Bogdanov, A.; Kaneva, E.; Shendrik, R. New insights into the crystal chemistry of elpidite, $\mathrm{Na}_{2} \mathrm{Zr}\left[\mathrm{Si}_{6} \mathrm{O}_{15}\right] \cdot 3 \mathrm{H}_{2} \mathrm{O}$ and $\left(\mathrm{Na}_{1+y} \mathrm{Ca}_{x} \square_{1-\mathrm{x}-\mathrm{y}}\right)_{\Sigma=2} \mathrm{Zr}_{2}\left[\mathrm{Si}_{6} \mathrm{O}_{15}\right] \cdot(3-\mathrm{x}) \mathrm{H}_{2} \mathrm{O}$, and ab initio modeling of IR spectra. Materials 2021, 14, 2160. [CrossRef] [PubMed]

61. Omori, K. Analysis of the infrared absorption spectrum of diopside. Am. Mineral. 1971, 56, 1607-1616.

62. Burns, R.G. Mineralogical Application of Crystal Field Theory; Cambridge University Press: Cambridge, UK, 1993. [CrossRef]

63. Goldman, D.S.; Rossman, G.R.; Dollase, W.A. Channel constituents in cordierite. Am. Mineral. 1977, 62, $1144-1157$.

64. Taran, M.N.; Langer, K. Electronic absorption spectra of $\mathrm{Fe}^{2+}$ ions in oxygen-based rock-forming minerals at temperatures between 297 and 600 K. Phys. Chem. Miner. 2001, 28, 199-210. [CrossRef]

65. Fontana, I.; Lauria, A.; Spinolo, G. Optical absorption spectra of $\mathrm{Fe}^{2+}$ and $\mathrm{Fe}^{3+}$ in aqueous solutions and hydrated crystals. Phys. Status Solidi B 2007, 244, 4669-4677. [CrossRef]

66. Abragam, A.; Bleaney, B. Electron Paramagnetic Resonance of Transition Ions; Oxford University Press: Oxford, UK, 2012.

67. Shongwe, M.S.; Al-Rahbi, S.H.; Al-Azani, M.A.; Al-Muharbi, A.A.; Al-Mjeni, F.; Matoga, D.; Gismelseed, A.; Al-Omari, I.; Yousif, A.; Adams, H.; et al. Coordination versatility of tridentate pyridyl aroylhydrazones towards iron: Tracking down the elusive aroylhydrazono-based ferric spin-crossover molecular materials. Dalton Trans. 2012, 41, 2500. [CrossRef] [PubMed]

68. Skogby, H.; Rossman, G.R. $\mathrm{OH}^{-}$in pyroxene; an experimental study of incorporation mechanisms and stability. Am. Mineral. 1989, 74, 1059-1069.

69. Burt, D.M. Metasomatic zoning in Ca-Fe-Si exoskarns. In Geochemical Transport And Kinetics; Hofmann, A.W., Giletti, H.S., Yoder, H.S., Jr., Yund, R.A., Eds.; Carnegie Institution of Washington: Washington, DC, USA, 1974; Volume 634, pp. $287-293$.

70. Pertsev, N.N. Skarns as magmatic and as postmagmatic formations. Int. Geol. Rev. 1974, 16, 572-582. [CrossRef]

71. Burt, D.M. Mineralogy and petrology of skarn deposits. Soc. Ital. Mineral. Petrol. Rend. 1977, 33, 859-873.

72. Meinert, L.D.; Dipple, G.M.; Nicolescu, S. World skarn deposits. Econ. Geol. 2005, 100, 299-336. [CrossRef]

73. Einaudi, M.T.; Burt, D.M. Introduction; terminology, classification, and composition of skarn deposits. Econ. Geol. 1982, 77, 745-754. [CrossRef]

74. Meinert, L.D. Skarns and Skarn Deposits. Geosci. Can. 1992, 19, 145-162.

75. Alaminia, Z.; Mehrabi, B.; Razavi, S.M.H.; Tecce, F. Mineral chemistry, petrogenesis and evolution of the Ghorveh-Seranjic skarn, Northern Sanandaj Sirjan Zone, Iran. Mineral. Petrol. 2020, 114, 15-38. [CrossRef] 